\title{
Role of quantum chemical calculations in molecular biophysics with a historical perspective
}

\author{
Alexander K. Kukushkin \\ Faculty of Physics, M.V. Lomonosov Moscow State University, \\ Vorobjevy Gory, 119992 Moscow, Russian Federation \\ Karl J. Jalkanen \\ Bremen Center for Computational Materials Science (BCCMS), University of Bremen, \\ Am Fallturm 1, D-28359 Bremen, Germany \\ and \\ Nanochemistry Research Institute (NRI), Curtin University of Technology, \\ GPO Box U1987, Perth WA 6845, Australia \\ and \\ Department of Molecular Biophysics, German Cancer Research Center [Deutsches \\ Krebsforschungszentrum] (DKFZ), Im Neuenheimer Feld 580, D-69120 Heidelberg, Germany
}

\begin{abstract}
We discuss how the basic principles of quantum chemistry and quantum mechanics can be and have been applied to a variety of problems in molecular biophysics. First, the historical development of quantum concepts in biophysics is discussed. Next, we describe a series of interesting applications of quantum chemical methods for studying biologically active molecules, molecular structures and some of the important processes which play a role in living organisms. We discuss the application of quantum chemistry to such processes as energy storage and transformation, and the transmission of genetic information. Quantum chemical approaches are essential to comprehend and understand the molecular nature of these processes. To conclude our work, we present a short discussion of the perspectives of quantum chemical methods in modern biophysics, the field of experimental and theoretical chiral vibrational and electronic spectroscopy.
\end{abstract}

\section{INTRODUCTION: HISTORICAL NOTES ON QUANTUM IDEAS AND CONCEPTS IN BIOPHYSICS}

This introduction is aimed at giving a short review of the development of quantum mechanical and chemical ideas and concepts in molecular biophysics. The formulation of the basic postulates of quantum theory was followed by publications discussing possible applications of this new theory to biology. Niels Henrik David Bohr and Erwin Rudolf Josef Alexander Schrödinger played a significant role in these discussions. In August of 1932 Bohr presented the public lecture at the International Congress of Light Therapists in Copenhagen in which he examined the significance of the Principle of Complementarity for understanding the fine organization of living organisms. This lecture was first published in "Nature" in 1933 
Role of quantum chemical calculations in biophysics with a historical perspective

[1]. Bohr wrote that, at a first glance, the essentially statistical nature of atomic phenomena contradicts the strikingly fine organization of living organisms. However, he found the properties of atomic processes that classical mechanics fails to describe, that find their place only within the complementary mode of description, to be as important for describing the behavior of living organisms as they are for describing the specific properties of inorganic matter. As an example of this, he cites carbon assimilation by plants, on which nutrition of animals so strongly depends. To understand these phenomena one has to deal with and understand the individual steps of the photosynthetic processes. Analogously, the non mechanical stability of atomic structures is clearly exhibited in the properties of chlorophyll and hemoglobin, very complex chemical compounds playing fundamental roles in the mechanics of assimilation in plants and in animal respiration, respectively [1,2]. Clearly, as early as 1932, Bohr imagined how the biophysics of photosynthesis would have to be described with the use of quantum theory.

Schrödinger devoted a book [3] to the relations between physics and biology. This book was based on the lectures he gave in February of 1943 in Dublin. In this work he discussed heredity problems in the context of quantum mechanics [3,4]. Quantum mechanics was the first theoretical theory which accounted from first principles for the vast variety of possible aggregates/complexes of atoms and molecules actually encountered in Nature. Consequently, one may safely assert that there is no alternative for the molecular explanation of the heredity substance. In fact, these predictions helped stimulate future progress and advances in molecular biology by focusing on the quantum molecular nature of biology. This stimulated the investigation of biological processes both experimentally and theoretically at the molecular level, which of course required and still requires the development of better tools, methods and instruments.

In 1935, while studying mutagenesis in Drosophila exposed to ionizing radiation, TimofeeffRessovsky, Zimmer, and Delbrück [5] determined the size of genes (as the size of the mutation regions) and hypothesized that genes are simple physicochemical units [5]. Frank and Teller [6] applied quantum mechanical ideas to study energy migration and photochemical action of solar energy absorbed in photosynthesis. Jordan [7] treated biological macromolecules as orderly organized solids and deduced their properties from the energy band structure. Using these considerations, he explained the action spectrum of photo inactivation of the enzyme urease and the mechanisms of gene interaction and mutation. Further progress in the adoption of the ideas and tools of atomic physics in biophysics was largely due to the works of Pauling and Corey [8] , Watson and Crick [9], Rosalind E. Franklin and R.G. Gosling [10,11] in which X-ray crystallography helped decipher two of the most important biological structures, the $\alpha$-helical structure of proteins and the double helical structure of DNA. Just a decade before, Szent-Gyorgui [12] suggested that proteins have the properties of semiconductors. This suggestion was followed by calculations of the protein energy spectrum made by Laki (cited from [13]) and Evans and Gergely [14]. This was the beginning of the second period (1955-1984) of spreading quantum concepts throughout the life sciences.

Experimental and theoretical methods of quantum physics were increasingly imported into biochemistry, biophysics, and molecular biology and became routine tools. The various optical methods (absorption spectroscopy, fluorescence spectroscopy, and Raman scattering) and radio spectroscopic techniques (electron spin resonance and nuclear magnetic resonance) were used in studies of the structure and properties of biologically important molecules. When the energy levels of biological molecules needed to be calculated, researchers used a simple quantum mechanical method of molecular orbitals proposed by Hückel (see [15]). The results achieved with this method were described in the monograph "Quantum Biochemistry" by B. Pullman and A. Pullman [15]. Later, molecular calculations were mostly done with the use of approximate methods based on the Roothaan and Roothaan-Hall equations. A comprehensive overview of quantum mechanical approaches in molecular biology can be found in the proceedings of a symposium [4] held at the 
Role of quantum chemical calculations in biophysics with a historical perspective

Laboratory of Biophysics of Stanford University, March 1963, and in the collections of papers "Theoretical Physics and Biology" [16].

Significant advances in the theory of optical spectroscopy were made in 1956 when Moffitt [17] developed a theory of the exciton spectrum of absorption for $\alpha$-helical polypeptides, which was based on Davydov's exciton theory of light absorption in molecular crystals [18]. Calculations of the exciton spectra correctly described the splitting of the 190-nm band and the optical activity of $\alpha$ helical structures [17]. In the early 1960s, Yomosa [4,19] and Suard, Berthier, and Pullman [20] used the self-consistent field approach to calculate (in a $\pi$-electron approximation) the electronic structure and the energy spectrum for the peptide group and for a polypeptide with H-bonds. The calculated results agreed well with the experimental spectroscopic data (cited from [4]), but were inconsistent with the theoretical estimates for the thermal energy of activation of electric conduction in proteins made by Cardew and Eley [21]. Their calculations abated the vigorous debate over the semiconductor properties of proteins, which were then thought to be essential for understanding the mechanism of enzyme catalysis in redox processes $[16,20,21]$.

It was during this time that electron spin resonance (ESR) spectroscopy, a method based on quantum physics, founds its way into biology. Biological applications of ESR received comprehensive coverage in the collection of papers entitled "Free Radicals in Biological Systems" [22], which included selected articles from the proceedings of a symposium held at Stanford University in March of 1960. To our knowledge, the ESR signal from a biologically important substance was obtained for the first time in 1954 by Commoner, Townsend, and Pake [23] in experiments with various lyophilized specimens of biological origin. When they began their work, only a low-sensitive spectrometer was at their disposal, and they could not then study specimens without subjecting them to lyophilization. A variety of tissues were studied: plant leaves and roots, muscle, brain, liver, lung tissue, myocardium, kidneys, and frog eggs. For all specimens, the concentration of free radicals was determined per gram of dry weight. Free radicals appeared to be most abundant in tissues with high metabolic rates, such as green leaves, liver, and renal tissue. Numerous research papers followed this pioneering study. Gordy et al. [24] recorded ESR signals from irradiated amino acids and proteins. Ehrenberg [25] and Beinert (cited from [22]) described ESR spectra of model semiquinones and flavin-dependent enzymes. Free radicals were detected in live, functional plant and animal cells $[26,27,28]$. Overall, those studies produced new insights into the mechanisms of redox processes in mitochondria and chloroplasts [29].

In the early 1960's, low-temperature radiospectroscopic studies of photosynthetic objects provided evidence of the electronic nature of the primary events in photosynthesis. In this context, of particular interest, is a study by Sogo et al. [28] reporting a result that came as a surprise. It turned out that the rise time of the ESR free radical signal recorded upon illumination of chloroplasts at room temperature was approximately equal to that at $-140^{\circ} \mathrm{C}$. This observation suggested the purely electronic nature of this signal, obviously unrelated to diffusion-controlled processes. Two years later, Sogo et al. [29] showed that the ESR signal at $g=2.0025$ detected previously [28] in Rhodospirillum rubrum chromophores could be also observed at $77 \mathrm{~K}$. This fact was thereafter confirmed with optical methods. Arnold and Clayton [30] described reversible changes in absorption at $420 \mathrm{~nm}$ during illumination of Rhodobacter sphaeroides chroloplasts at $1 \mathrm{~K}$. Soon afterward, Chance and Nishimura [31] detected temperature-independent cytochrome oxidation in the purple photosynthetic bacterium Chromatium and then determined the cytochrome oxidation rate as a function of temperature down to $30 \mathrm{~K}$ [32].

In their study of 1966, DeVault and Chance [32] interpreted the accumulated data, suggesting a tunneling mechanism for electron transfer from cytochrome to bacteriochlorophyll in Chromatium, 
and estimating the rate of this process using the known formula for the probability of a quantum particle tunneling through a barrier. This was the first evidence for the existence of a purely quantum mechanical effect in photosynthesis (in addition to the photon absorption by the pigments). After that study by DeVault and Chance [32], many theoretical descriptions of the experimentally obtained temperature dependence of the electron transfer rate were developed that employed and made use of various assumptions, see for example, Chernavsky et al. [33,34], Hopfield [35], Jortner et al. [36,37,38], Dogonadze, Kuznetsov and Ulstrup [39], Petrov et al. [40], and Sarai [41]. For reviews of studies addressing the basis of electron transfer in biological systems, see Jortner [42] and Petrov [13].

Yet another phenomenon that was discovered during this period was damped oscillations. Experiments showing damping oscillations in the oxygen flash yield with flash-illuminated plant preparations, the oscillation period being equal to four flashes, needed to be explained and understood [43,44]. To explain this phenomenon, Kok et al. [45] put forth a hypothesis that the water-splitting oxygen-evolving system in plants can exist in different discrete states. By that time, picosecond techniques made it possible to study the temporal characteristics of the events involved in the primary charge separation in bacterial reaction centers $[46,47]$. The data obtained provided evidence that quantum transitions between discrete states take place during photosynthesis.

More recently, the photosynthetic reaction center from the purple bacterium Rhodopseudomonas viridis was determined by new crystallographic techniques by Deisenhofer et al. [48]. The bacterial reaction center became the first of the protein complexes of photosynthetic membranes whose structure was known at a resolution of $3 \AA$. This was a major breakthrough in the field. Knowledge of the spatial structure of the photosynthetic reaction center resulted in new problems and questions coming to the forefront. Their solution brought interesting and unexpected results. First, in the course of very complicated femtosecond spectroscopy measurements, a fast process of electron transfer from the excited primary electron donor to the primary acceptor was detected. Its time constant was estimated at 3 ps at room temperature. Moreover, on cooling down to10 K, this process becomes 1.5-2.0 times faster [49,50,51]. Second, shortly thereafter, quantum oscillations of electron donor fluorescence in the spectral region from 695 to $930 \mathrm{~nm}$ were revealed by Vos et al. [52,53,54] in the bacterial reaction center, which have a period of $260 \mathrm{fs}$.

These findings caused a flow of theoretical studies in which quantum mechanical approaches were used to describe the characteristics of electron transfer obtained in experiments. For example, the explanations given by Bixon [55] and Michel-Beyerle et al. [56] were based on the Marcus theory of the electron transport [57]. They quantitatively described the mechanism of charge separation in the bacterial reaction center. Specifically, they derived a formula for the rate of electron transfer and provided a rationale for why this process is unidirectional. The term unidirectional is commonly used to describe a fast electron transfer along the electron carrier chain within the protein subunit $\mathrm{L}$ of the reaction center. According to Michel-Beyerle et al. [56], this effect is accounted for by an interaction of electron donors and electron acceptors with polar groups of the proteins and by asymmetry (in the sense of $\mathrm{M}$ and L chains) of polarizing Coulomb interactions. The electrostatic control of electron transfer in the bacterial reaction center was also addressed by Parson et al. [58]. Molecular displacements during charge transfer were computed by standard molecular dynamics methods [59]. Using the density matrix operator, Mukamel and associates [60] made calculations of electron transfer by superexchange between the electron donor and the primary acceptors in the bacterial reaction center. A vast literature on various aspects (including quantum ones) of electron transfer in the reaction center was reviewed in Shuvalov's book [61]. 
Role of quantum chemical calculations in biophysics with a historical perspective

\section{QUANTUM CHEMICAL PROPERTIES OF BIOLOGICALLY IMPORTANT SUBSTANCES}

Biophysical studies applying quantum chemical methods can have various foci. There are works which focus on the electronic properties of biological substances for an explanation of the experimental data, those which focus on the correlation between the structure and biological activity, and finally those which focus on understanding biophysical processes occurring at the molecular and submolecular level.

\section{A. Quantum mechanical calculations for proteins, peptides and model systems}

Calculations of the electronic properties of proteins relate to the semiconductor hypothesis concerning proteins [10] and to the experiments of Cardew and Eley [21]. First, Evans and Gergely studied the $\pi$-electron structure of a network consisting of peptide groups [14]. They used the linear combination of molecular orbitals (LCAO) Hückel molecular orbital (HMO) approximation, and assumed that the $\pi$-electron system was composed of $2 \mathrm{p}_{\mathrm{z}}$-orbitals of carbon, oxygen and nitrogen atoms. The energy levels obtained were classified into three bands of width of approximately $0.1-0.3 \mathrm{eV}$. The lowest bands were occupied by electrons and the highest orbital was empty. The values of the energy gap between the highest occupied molecular orbital (HOMO) and lowest unoccupied molecular orbital (LUMO) [highest occupied and lowest unoccupied bands], the so-called HOMO - LUMO gap, were calculated to be $3.05 \mathrm{eV}(410 \mathrm{~nm})$ and $4.23 \mathrm{eV}(290 \mathrm{~nm})$ for the cases of trigonal (tr) and pyramidal (pyr) symmetry of the nitrogen atom, respectively. The width of the bands depended on the resonance integral value $\beta_{\mathrm{N}}$ between the neighboring peptide groups, which is very sensitive to the length of the H-bonds. Because of the very small value of $\beta_{\mathrm{NO}}(-0.2 \mathrm{eV})$, it may be expected that the centers of the bands are approximately equal to the energies of three molecular orbitals of the isolated peptide group (in eV); $\varepsilon_{1}=-15.99(\operatorname{tr} \mathrm{N}),-16.8 \mathrm{eV}\left(\right.$ pyr N); $\varepsilon_{2}=-12.77,-14.03 \mathrm{eV}$; and $\varepsilon_{3}=-9.39$ and $9.60 \mathrm{eV}$, respectively. These results were supported by the experimental investigation of the temperature dependence of the electroconductivity by Cardew and Eley [21]. The values of the HOMO - LUMO gap calculated for glycine, polyglycine and hemoglobin were $2.92 \mathrm{eV}, 3.12 \mathrm{eV}$, and $2.75 \mathrm{eV}$, respectively. However, the predicted optical absorption in the region of 290 and $410 \mathrm{~nm}$ for diglycine, triglycine or polyglycine was not seen in the experimental absorption spectra [21].

The contradictions between the optical spectral simulations and the results of Cardew and Eley's experiments demanded new and better quantum chemistry calculations. Such calculations were carried out using more advanced methods in France by Suard, Pullman and Berthier $[20,62,63]$. In these works the study on the electronic structure of proteins has been carried out for the determination of the width and positions of the energy bands produced by the interaction of the $\pi$-electron systems of the peptides through H-bonds. In doing so, the isolated peptide group has been considered as a six-electron system, including the four conjugated $\pi$ electrons of CONH peptide moiety and the perpendicular lone-pair $(n)$ of the carbonyl oxygen. Two peptide groups connected through an H-bond are thus represented as a twelve-electron system and so on. The method applied in that work was the self-consistent treatment of Roothaan [64], in which the approximations of Pariser and Parr [65] have been used in an improved manner [20] with the help of a Löwdin's orthogonalized set of atomic orbitals [66]. The main conclusions from this work are the following. The energy band width obtained by an SCF treatment is considerably larger than that obtained by Evans and Gergely [14] (of the order of $1 \mathrm{eV}$ versus $0.25 \mathrm{eV}$ in the Hückel approximation). The lowest transition energy shows only very small variations as the number of peptide units increases. This result corresponds to the lack of an observable displacement of the longest wave length of absorption in proteins with respect to that of monopeptides [67]. The energy gap for the singlet-singlet transition is of the order of $5 \mathrm{eV}$, a result which seems to forbid the possibility of an intrinsic semiconductivity in proteins.

A similar investigation of the electronic structure of peptides and proteins was carried out by Yomosa [19]. Using the zero-integral-overlap approximation within the Roothaan method the energies of the one electron molecular orbitals were (in eV): $\varepsilon_{1}=-15.34 ; \varepsilon_{2}=-11.25 ; \varepsilon_{3}=-0.93$. The excited states of the peptide group had energies: ${ }^{1} \mathrm{E}_{2 \rightarrow 3}=6.672 ;{ }^{3} \mathrm{E}_{2 \rightarrow 3}=2.603 ;{ }^{1} \mathrm{E}_{1 \rightarrow 3}=10.058 ;{ }^{3} \mathrm{E}_{1 \rightarrow 3}=6.937$. By introducing an interaction 
Role of quantum chemical calculations in biophysics with a historical perspective

between these configurations, the energies of the excitations (in eV) were: ${ }^{1} \mathrm{E}_{-}=6.547(189.5 \mathrm{~nm}) ;{ }^{1} \mathrm{E}_{+}=$ $10.182(121.8 \mathrm{~nm}) ;{ }^{3} \mathrm{E}_{+}=2.306 ;{ }^{3} \mathrm{E}_{-}=7.234$.

The experimental absorption data gives values of $197 \mathrm{~nm}$ for dimethylformamide and $185 \mathrm{~nm}$ for glycine, respectively. Thus sufficient agreement exists between the calculated and experimental values of the oscillator strengths of the corresponding transitions. In the work of Yomosa the energy structure of the infinite crystal of peptide groups bonded by H-bonds were calculated. The width of the first triplet exciton zone with center near $2.58 \mathrm{eV}$ turned out to be equal to $10^{-5} \mathrm{eV}$, the second singlet zone (the centre near 6.65 $\mathrm{eV}$ ) to be $0.47 \mathrm{eV}$; and the zone of conductivity (near $10.2 \mathrm{eV}$ ) to be $0.21 \mathrm{eV}$. These data clearly showed that the intrinsic conductivity of proteins is impossible. Subsequently, the authors of experiments [21] were shown not to have taken into account some complicated experimental circumstances such as ionic and surface conductivity of the samples. Later on, calculations taking into account the $2 p$ and $3 \mathrm{~d}$-orbitals of the peptide group [13] were carried out. However, it is necessary to note that for these calculations the investigators did not take into account the polarization of the surrounding media by the excess charge. Taking these effects into account could change the position of conductivity zone. Lyons has pointed out the necessity to take into account the polarization effects for studies of organic molecular crystals [68]. The quantitative estimation of the energetic spectrum of the polar states for organic crystals of conjugated hydrocarbons was fulfilled by Blumenfeld and Benderskiy [69]. The spectrum of the polar states for a network of peptide groups was calculated in [70]. From these estimates it was determined that the energy level corresponding to conductivity zone has an energy $(\Delta \mathrm{E}=3.1 \mathrm{eV})$ greater than the energy of the ground state of the system. This is smaller than the gap determined by Yomosa [19]. Recently, it has become apparent that the next problem to address is how to best determine and take into account the role of the protein surroundings on the electronic properties in the primary photosynthetic processes under low temperatures [71] and also later, at room temperature, where things get even more complicated.

\section{B. The quantum chemistry studies of the nucleic acids}

The quantum chemical studies of nucleic acids may be divided into three parts. In the first part the scientists have studied the electronic and optical properties of the components of native nucleic acids. One important example is Tinoco's paper "Hypochromism in Polynucleotides" [72]. In this paper the author proposed the first explanation of hypochromism where the native DNA has about 30\% less absorption at 260 $\mathrm{nm}$ than the denatured sample.

Tinoco supposed that it takes place because of the energy migration between the adjacent pairs of bases. This phenomenon is explained by the anisotropy of tensor for the dipole-dipole interaction. Using perturbation theory, he gave the following formula for hypochromic effect:

$$
\frac{F_{o a}}{f_{o a}}=1-\frac{k \lambda_{o a}^{2} f_{o a}}{N} \sum_{i=1}^{N} \sum_{j \neq i}^{N} G_{i j} \vec{e}_{i} \vec{e}_{j}-\frac{4 k \lambda_{o a}^{2}}{N} \sum_{a^{\prime} \neq a} \sum_{i=1}^{N} \sum_{j \neq i} \frac{G_{i j} \vec{e}_{i} \vec{e}_{j} f_{o a^{\prime}} \lambda_{o a^{\prime}}^{2}}{\lambda_{o a}^{2}-\lambda_{o a^{\prime}}^{2}}
$$

In this formula the author used the notations.

$\mathrm{F}_{0 \mathrm{a}}$ - the oscillator strengths for polymer per one chromophore in absorption band $0 \mathrm{a}$.

$\mathrm{f}_{\mathrm{oa}}$ - the oscillator strengths for monomer chromophore in absorption band 0a.

$$
f_{o a}=\frac{2303 m c^{2}}{\pi e^{2} N_{0}} \int \varepsilon_{o a} d \omega=4.32 \cdot 10^{-9} \int \varepsilon d \omega,
$$

$\mathrm{k}$ is a constant value, which equals $3 \mathrm{e}^{2} / 8 \pi^{2} \mathrm{mc}^{2}$.

$\lambda_{0 \mathrm{a}}$ - the wavelength $\left(\mathrm{A}^{0}\right)$ of absorption maximum for monomer in bands $0 \mathrm{a}$. 
$\mathrm{G}_{\mathrm{ij}}$ - the tensor dipole-dipole interaction.

$$
G_{i j}=\left[\vec{e}_{i} \vec{e}_{j}-\frac{3\left(\vec{e}_{i} \vec{r}_{i j}\right)\left(\vec{e}_{j} \vec{r}_{i j}\right)}{\vec{r}_{i j}^{2}}\right] \frac{1}{/ \vec{r}_{i j} / 3}
$$

$\vec{e}_{i}, \vec{e}_{j}$ - the unit vectors in the directions of the transition moments in the chromophores i and $\mathrm{j}$.

$\vec{r}_{i j}$ - the distance between centers of chromophores $\mathrm{i}$ and $\mathrm{j}$.

Tinoco applied this formula to calculate the hypochromic effect for native DNA. Based on the formula, Tinoco calculated the value of $\mathrm{F}_{0 \mathrm{a}} / \mathrm{f}_{0 \mathrm{a}}$ as function of the angle $\varphi$ between the axis of symmetry of the bases and the direction of $\mathrm{H}$-bonds in the base pair under consideration. At the same time the angle $\varphi$ changed from zero to $\pi$. At $\varphi \approx 60^{\circ}$, the ratio of the oscillator strengths becomes equal to 0.7. This corresponds to a hypochrome effect of about $30 \%$. This value coincides with the one determined for native DNA. The derived function permits one to determine the direction of the dipole transition moment lying in the plane, which is perpendicular to the DNA axis.

Subsequently a similar approach was applied by Rhodes [73]. Applying first-order perturbation theory, he derived an expression which relates the hypochromism to the geometry of the transition moments of the mononucleotide residues in the polymers. Additionally, the hypochromism of biopolymers was considered by DeVoe [74]. He presented a local-field treatment of hypochromism that is physically equivalent to TinocoRhodes consideration, but which may be easier to introduce intuitively. In addition, DeVoe studied the contribution of the solvent to hypochromism. It may be noted that Nesbet [75] and Bolton and Weiss [76] pointed out that Tinoco and Rhodes derived their equivalent hypochromism formulas by assuming strong coupling, which is not present in DNA. Going back to the original work of Kirkwood [77] Tinoco corrected both his formulas as applied to polymers [78] and polynucleotides [72]. See the erratum of references [72] and [78] for the complete set of original equations and the newly derived/corrected equations. We have presented the original equation in equation (1). The correction in [72] is as follows:

$$
\frac{F_{o a}}{f_{o a}}=1-\frac{4 k \lambda_{o a}^{2}}{N} \sum_{a^{\prime} \neq a} \sum_{i=1}^{N} \sum_{j \neq i} \frac{G_{i j} \vec{e}_{i} \vec{e}_{j} f_{o a^{\prime}}^{\prime} \lambda_{o a^{\prime}}^{2}}{\lambda_{o a}^{2}-\lambda_{o a^{\prime}}^{2}}
$$

The quantum chemistry properties of the nucleic acids and their constituents were studied and discussed by B. Pullman and A. Pullman [79]. They used conventional Hückel theory to calculate the electronic characteristics of biologically important molecules. Subsequently, Veillard and Pullman [80] produced $\pi-$ electron calculations using the semi-empirical antisymmetrized molecular orbital (ASMO) self-consistent-field (SCF) method [81,82]. Later, Bradley, Lifson and Honig [83] used united Veillard-Pullman $\pi$ charges with $\sigma$ charges determined by the method of Del Re [84] to get a set of net charges summarizing both $\sigma$ and $\pi$ contributions. To eliminate the shortcomings in these methods Rein et al. [85] proposed the iterative extended Hückel method which led to further improvement.

Using this method Rein et al. [85] calculated atomic charge distributions for nucleotide bases, dipole moments and the orbital energies of the lowest unoccupied molecular orbital and highest occupied molecular orbitals. Analyzing the energy of highest occupied $\pi$ orbitals of nucleotide bases, the authors noted that the highest occupied orbital was not always of $\pi$ symmetry.

Although some interesting and exciting electronic properties of nucleic acids have been obtained using the Hückel method, there still exists some non-uniqueness due to choice of the parameters used in this and other semi-empirical methods. This can be seen by comparing the results of the various works $[86,87]$ where different sets of parameters were used. Moreover, some properties of nucleic acids, such as the electronic absorption spectra (the energy of the transitions in triplet states), require the application of more accurate 
Role of quantum chemical calculations in biophysics with a historical perspective

methods, for example, the self-consistent field (SCF) methods: unrestricted Hartree-Fock (UHF), time dependent density functional theory (TDDFT); and finally Greens functions integral approaches.

Many such studies were undertaken by a strong group of Ukrainian scientists [88,89,90,91,92,93]. In these papers the following problems were attacked. In [88], Danilov et al. studied the $\pi$-electron structure the Watson-Crick base pair by the methods of the self-consistent field in the Parisier-Parr-Pople (SCF-PPP) approximation $[81,82]$. The ground and excited states were investigated. The authors determined the H-bond contribution to the energy of the base pair, and the energies of singlet and triplet transitions. Moreover, the charge transfer states were discussed in connection with the proton tunneling in DNA and problems involved in mutations, aging and tumors [94]. These results were discussed in the light of proton transfer along $\mathrm{H}$ bonds.

In [89], the tautomerization of bases was investigated using the SCF-PPP approximation. The authors estimated the rate constant for tautomeric transformation. They came to the conclusion that transformation is possible only for the excited states of base pairs. In [90], Danilov et al. considered the electronic aspects of photodimerization of the nucleic acid bases. As is well known, the ultra violet irradiation of bacteria and phages brings about mutations and lethal destruction. As a rule, the mutations are composed of transition from G-C to A-T and possibly deletions and insertions. On the molecular level these processes are explained by the intra- and intermolecular photodimerization. It is known [95] that the photodimerization of the bases takes place in the triplet states of bases. That's why the authors of this paper carried out the calculations studying the electronic characteristics of the pyrimidine bases in their lowest excited states. They used the SCF method for open shell systems because using this method one obtains different wave functions for the excited singlet and triplet states. Moreover, this method permits one to calculate the characteristics of the ground and excited states in the identical approximation. The authors calculated the $\pi$-bond orders for the lowest triplet states of thymine (T), uracil (U) and cytosine (C), and in addition, the bond orders and the electron density on the atoms $\mathrm{C}_{5}$ and $\mathrm{C}_{6}$ were determined (See Figure 1 below for atom numbering, reproduced from [91], same number scheme as used in Fig 1 \{A. Pullman, (1969) Annals New York Academy of Sciences 158:65-85\} [96] and in Fig 4.4 \{Benjamin Lewin (1991) Gene, Lehrbuch der molekularen Genetik, Zweite Auflage, VCH, Weinheim, page 65\}[97]). It turned out that in the triplet state, the bond order of the $\mathrm{C}_{5}-\mathrm{C}_{6}$ bond strongly diminishes and favors the dimerization reaction [98]. This is consistent with UV damage to DNA via dimerization, which is repaired by the photolyase class of proteins in the presence of blue light. The energy of the first singlet transition for thymine was found to be $4.71 \mathrm{eV}$, which is in good agreement with the experimental result.

\section{Insert Figure 1 here}

In [91] the $\pi$-electron structure of the base pairs was calculated using a semi-empirical SCF method; more precisely, the energetic characteristics of the ionized states of the base pairs were calculated. The values of the ionization potential (IP) and electron affinity (EA) were calculated by using the wave functions for the cation and anion constructed from the molecular orbitals for the ground and excited state orbitals obtained using open shell methods. The authors calculated the values of the IPs and the EAs for adenine (A), A-T and guanine (G), G-C base pairs and the $\pi$-electron density on all of the atoms.

These results demonstrated that the electron donating and electron accepting capabilities of the G-C pair are larger than those of the A-T pair. A comparison of the electron density in the cationic and ground states showed that in the transition to the cationic state the electron density changes mainly in the purine bases on atoms $\mathrm{C}_{5}$ and $\mathrm{C}_{8}$ of the $\mathrm{A}-\mathrm{T}$ and the G-C pairs, respectively. This means that the electron donor of the A-T pair is an adenine, and the electron donor of G-C pair is a guanine. Considering the anionic and ground states, one can see that the acceptors of the electrons are the pyrimidine bases. In this case the largest change of the electron density takes place on the $\mathrm{C}_{14}$ and $\mathrm{C}_{16}$ atoms of the $\mathrm{A}-\mathrm{T}$ pair, and on the $\mathrm{C}_{15}$ and $\mathrm{C}_{17}$ atoms of G-C pair, respectively. See atoms identification in [91]. During the transition of the base pairs from the unionized to the ionized state, the essential changes in the electron density takes place on the proton-donor and protonacceptor atoms of the same H-bonds. In doing so, these changes bring about an alteration/change in the form of proton potential curve and, consequently, a change in the proton transfer process along the corresponding $\mathrm{H}$ bond, see for example the hypothesis due to Löwdin [94]. 
Role of quantum chemical calculations in biophysics with a historical perspective

In [93] the electronic structure of the DNA base pairs in the excited states was considered relative to Löwdin's hypothesis about the mechanism of mutations [94]. The history of this question is the following. Löwdin assumed $[94,99]$ that due to the absorption of ultra violet radiation by a DNA molecule it is possible that the rate constant for proton tunneling strongly increases. The changing proton position in the H-bond may be induced by alternation of the form of the proton potential energy curve because of redistribution of the electron density in the molecule. In $[100,101,102]$ the authors studied the electronic structure of the molecular state corresponding to the electronic transition from the HOMO to LUMO. It was shown that this state is the state with the charge transfer. The study of proton potential curve showed that in the excited state the rate constant of proton tunneling strongly increases. These results were used for the explanation of mutagenic effect of ultra violet light. However, in [88] it was shown that the lowest energetic state of system under consideration was not the state containing the transition $n \rightarrow n+1$, but the state in which the excitation is localized on the one of the two bases. It turns out that the results in $[100,101,102]$ are related to higher excited levels; the authors investigated the electronic structure of the excited triplet and singlet states by the semiempirical antisymmeterized molecular orbital (ASMO) self-consistent-field (SCF) method taking into account the interaction of 24 singly excited configurations. For the $\mathrm{T}_{1}$ state of two pairs the excitation completely localized on the pyrimidine base, namely on the $\mathrm{C}_{14}-\mathrm{C}_{15}$ bond. In so doing the electron densities on the donor and acceptor proton atoms practically do not change. The phenomenon also takes place for the $S_{1}$ state pair for A-T and G-C. That's why the excitation of these pairs does not bring about an increasing rate constant of proton tunneling. The excited singlet and triplet states of the adenine-thymine and guanine-cytosine base pairs were estimated using the configuration interaction method and the SCF open shell method in a semi-empirical approximation [92]. The determined values of the singlet-singlet transitions agreed with the experimental results, and it was shown that the forming of a pair from separate bases does not lead to an essential change in the strength of the first absorption band and therefore can not explain the hypochromic effect in DNA. The value of the transfer charge for the first singlet excited state is calculated to be 0.1 for A-T pair and 0.18 for G-C pair (in the electron charge units), respectively. The charge transfer in the lower triplet state is negligibly small. The participation of the first excited singlet and triplet states in proton tunneling appears to be a highly unlikely mechanism for photo-induced mutation.

The calculations of ground state potential energy curves for the nucleic bases relative to the problems of tunneling and tautomeric equilibrium were made by several groups of workers [103,104]. However, the results obtained by the different approximations were not always in agreement. Using a semi-empirical SCF approximation Rein and Harris [103] have found a double well potential in both a model that did not include the possibility of tautomeric displacements in the two $\mathrm{N}-\mathrm{H}--\mathrm{O} \mathrm{H}$-bonds, and in a model which involves simultaneous rearrangements involving more than one H-bond of the guanine-cytosine base pair. Analogous results were obtained by Blizzard at al. [105]. By applying an ab initio method it was later found that a calculation which contains only one H-bond turns out to yield no double-well [104]. These contradictions were considered by Maranon and Sorarrain in [106]. The calculations were made using the CNDO/2 approximation. The calculations were performed with the proton placed at various points along the axis of their respective $\mathrm{H}$ bonds. In order to consider the effects of cooperativity on the doubly H-bonded structure, the computations were first previously restricted to nuclear configurations which could arise from single displacement of the proton under consideration and then simultaneous displacements of both protons were analyzed. The results of this work are as follows. In the first case, for the single displacement of the two protons there is not a double well potential curve. The changes of the potential energy for the simultaneous displacement of proton of two $\mathrm{H}$-bonds between adenine and thymine do have double well character. The height of the barrier was $3.75 \mathrm{eV}$. The analysis of the structure of the eigenvectors involved in the observed optical transitions has shown that the $\pi$ lone-pair orbitals are perhaps the main contributors to the formation of the H-bonds. The estimation of the magnitude of the equilibrium constant between the normal and tautomeric forms $\left(\mathrm{K} \approx 10^{-32}\right)$ showed that the simultaneous double proton tunneling tautomerization is rare and negligible. That's why it is not a cause of error in the cellular reproduction.

The interesting results, received by the complete active space with second-order perturbation theory (CASPT2) method, based on the second-order Møller-Plesset perturbation theory (MP2) applied on a complete active space SCF (CASSCF) reference wave function, mean that the conical intersection of the energy hypersurfaces for the ground state and the $!\left(\pi \pi^{*}\right)$ state is responsible for ultrafast decay of singlet excited state 
Role of quantum chemical calculations in biophysics with a historical perspective

of cytosine [107]. In [108], it was shown that the fluorescence lifetimes of DNA and RNA nucleosides fall on the subpicosecond time scale. This is an efficient waste of energy in order to prevent photoreactions of unwanted lethal consequences.

Quantum chemistry calculations have played an important role in studying the structure of nucleic acid structure. In 1980's it was shown that the physical properties, conformations and the biological functions in many respects are determined by the non-bonded interactions [109]. The non-bonded interactions were calculated using atom-atom potential functions having the form:

$$
\begin{aligned}
& U\left(r_{i j}\right)=\left(e_{i} e_{j}\right) /\left(\varepsilon r_{i j}\right)-A_{i j} r_{i j}{ }^{-6}+B_{i j} r^{-12} \\
& U\left(r_{i j}\right)=\left(e_{i} e_{j}\right) /\left(r_{i j}\right)-A_{i j}(10) r^{-10}+B_{i j}{ }^{10} r^{-12}
\end{aligned}
$$

Formula (2) was used for calculating all the non-bonded atom-atom interactions except those nonbonded interactions between the atoms involved in H-bonds (the hydrogen atom and the hydrogen acceptor, either an oxygen or a nitrogen atom), for which formula (3) was used. In [110] the procedure of parameter choice $\left(\mathrm{A}_{\mathrm{ij}}\right.$ and $\left.\mathrm{B}_{\mathrm{ij}}\right)$ was proposed on the theoretical basis the experimental data about the types and energies of intramolecular interactions in the crystals and complexes of nucleic acids components. The atomic charges used in formulas (2) and (3) were obtained by quantum chemistry methods. The effective charges on atoms the authors $[109,110]$ have received by summation of the $\pi$ - electron charges, determined by the Hückel method [110], and $\sigma$-electron charges, calculated with help of parameter suggested by Berthod and Pullman [111]. By using these new non-bonded atom-atom potentials for calculating the non-bonded interactions, important and new results were obtained. Possible mechanisms involving intermediate mis-pairings errors in biosynthesis of nucleic acids were studied. Here one used non-bonded interaction energy calculations to investigate possible mis-pairings, to investigate possible conformational changes of the double helix within A- and B-families of nucleic acids, and to analyze the conformations of the nucleic acid fragments containing nucleotide mispairings. Finally the parameter and potential choice for calculation of the energy of non-bonded interactions in nucleic fragments was investigated. These results led to the use of a separate potential for the non-bonded interactions involving H-bonded atoms.

Subsequent researchers have also developed separate non-bonded potentials for calculating the nonbonded interactions for $\mathrm{H}$-bonding. What has been a real challenge is to be able to reproduce the cooperative effects involved in $\mathrm{H}$-bonding and the charge transfer. The dipole moment of molecular gas phase water molecules and bulk water molecules are very different. Hence Jorgensen and coworkers have developed the optimized potentials for liquid simulations (OPLS) force field. Other groups have tried to implement polarizable atoms in their force fields. A general theory which adequately treats these all of these effects for the both the ground and all excited electronic states has not yet been found. There are many different solutions to this problem, none being high general and transferable. There are many force fields developed for only water, and one much choose a potential to use not only for water, but also for all the bonded and non-bonded interactions for atoms within large biomolecules, between them for the non-bonded interactions, and finally also between the atoms in the molecules and the solvent. Experimental neutron diffraction data has shown that the water molecules near the protein and nucleic acid surface are different from bulk water molecules. Hence the possibility to have different kinds of water molecules in one's experiment and in one's simulation, and hence different potentials for different types of atoms. The whole concept of molecular mechanics force fields is based on being able to parameterize the intermolecular and intramolecular interactions. Quantum mechanics has been used for the intermolecular potentials, but has failed in determining the non-bonded potentials, due to an inability to accurately calculate the dispersion forces and interactions by Hartree-Fock and most density functional theory (DFT) level calculations. Here the experimentally determined non-bonded interaction potentials have been more accurate than those derived from theory. But one requires very good experimental data to derive the non-bonded potentials experimentally. This data is available for amides and proteins, but not for all types of molecules. With the recent development of ab initio DFT methods by the 
Role of quantum chemical calculations in biophysics with a historical perspective

Bartlett group [112], so-called optimized effective potentials (OEPs) for electron correlation, this may change. But to date, no systematic derivation of the non-bonded potentials for all of the various atom types used in molecular mechanics force fields with all other atoms has been undertaken. Clearly such work is very important. In addition, for heavy metals, relativistic calculations are required. Relativistic pseudopotentials have been developed for many heavy atoms, but not for all. And these relativistic pseudo potentials involve some parameterization, so one is able to reproduce with relative good accuracy the experimental data one has used in the parameterization. But as one has seen with molecular mechanics force fields and various exchange correlation (XC) functionals which did not include vibrational frequencies during their development, one can produce/derive a set of potentials, parameters and XC functionals which reproduce one or more property, but do pretty bad for other properties. Hence the problem is one of generalizability and transferability.

The main results of the early sub-molecular studies $[110,113,114,115,116]$ are the following. All types of base pairs in the tautomeric forms could be incorporated into the double helix with no short interatom contact arising and without drastic changes of the sugar-phosphate backbone. All known experimental data on spontaneous errors in nucleic acid biosynthesis could be explained by formation of such pairs

New insight into the nature of the electron spectra of nucleic acid bases came with the work of Hug and Tinoco [117]. Earlier explanations for the electronic properties of nucleic acid bases were based on separate $\sigma$ and $\pi$-electronic structure calculations. However, it is known that $\pi$-electron estimations do not take into account the polarization of the $\sigma$ core that is important in molecules containing several different hetero-atoms. In the early works one only used single Slater determinant descriptions for describing the excited states which included all valence electrons $[118,119]$. However, a single Slater determinant description of the excited states of benzene and other aromatic molecules is qualitatively insufficient. Hence in [117] the authors calculated the spectra of nucleic acid bases, their tautomers, and ions with an all valence electron SCF-MO method (CNDO-CI) that included configuration interaction for the excited states. As a basis set the authors used 1s orbitals for hydrogen atoms and 2s, 2p Slater orbitals for atoms in the first row. The singly excited configurations are obtained by using the virtual orbitals of the ground state calculation. The 120 configurations which are lowest in energy were used. It was shown that the spectra of pyrimidine and purine bases can be divided into two groups, namely the cytosine and uracil type and adenine and hypoxanthine type. Due to an additional proton at one of the nitrogen atoms of the six-membered ring, uracil and hypoxanthine type spectra differ from cytosine and adenine type spectra. In pyrimidine bases, the presence of this proton leads to an increase in intensity of the transition lowest in energy and a decrease in intensity and shift towards higher energy of the second level to lowest one. In purine bases the excited states are more similar to those of purine itself but distinctly different from does of the underlying indole system. Adenine-type spectra are characterized by the fact that the lowest transition is localized mainly at $\mathrm{N} 7=\mathrm{C} 8$ in the five-membered ring, whereas the transition spreads out over the fragment $\mathrm{C} 2=\mathrm{N} 3-\mathrm{C} 4=\mathrm{C} 5-\mathrm{N} 7=\mathrm{C} 8$ and corresponds to the lowest transition in triene. In hypoxanthine-type spectra these two transitions are interchanged. Protonation at N7 in the five-membered ring reverses this change and leads back to an adenine type spectra. The results of this article showed that not only $\mathrm{n} \rightarrow \pi^{*}$ transitions, but also $\pi \rightarrow \pi^{*}$ transitions, are profoundly affected by protonation and tautomerization.

The mispairing ability of a nucleotide base analogue, $\mathrm{N}^{4}$-aminocytosine, was considered in work [120] by an ab initio molecular orbital method. The structures of the isomers of $\mathrm{N}^{4}$-aminocytosine were optimized with the 3-21G basis set by use of gradient optimization techniques. This basis was used for the calculations of relative energies and dipole moments. $\mathrm{N}^{4}$-aminocytosine, a nucleotide analogue of cytidine, is a potent mutagen. It can be incorporated as a residue in DNA, causing transitions from AT to GC and from GC to AT. The calculations of the energies and dipole moments of isomers of $\mathrm{N}^{4}$-aminocytosine have shown that $\mathrm{N}^{4}$ aminocytosine might exist as any of the four isomers under influence of some other components in its vicinity. This possibility may be the origin of the mutagenic activity of $\mathrm{N}^{4}$-aminocytosine. In results of calculations it was shown that the G-C-like base pair becomes more stable than G-C base pair itself when $\mathrm{N}^{4}$-aminocytosine is incorporated instead of cytosine. However, an A-T-like base pair becomes less stable than A-T base pair itself when imino-E form of $\mathrm{N}^{4}$-aminocytosine is substituted for thymine. The authors remarked that this might be related to the effects of the incorporation of $\mathrm{N}^{4}$-aminocytosine into replication DNA. 
Role of quantum chemical calculations in biophysics with a historical perspective

Tarashima studied optical anisotropy of synthetic polynucleotides [121]. In [121] the $\pi$-electron polarizability of uracil and adenine was considered, and it was shown that the index of refraction in the visible and ultraviolet region plays an essential role in birefringence. Helical polynucleotides have been shown to have a negative birefringence. This means that the refractive index along the minor axis is larger than one along the major helical axis. The larger transverse polarizability of helical polynucleotides originates from the base pairs which are stacked with their molecular planes normal to the major helical axis. That's why the negative birefringence is a reflection of the base stacking. Moreover, polarizability plays a part in molecular interactions as a component of the van der Waals forces. In [122,123] it was shown that the stability of double helices originates from the stacking energy of bases rather than the H-bonds. The stacking energy is determined by dipole-dipole, dipole-polarizability, and polarizability-polarizability interactions. In [121], the Hückel method and the self-consistent-field (SCF) are used for the calculations of polarizability. The theoretical values obtained by the Hückel method are consistently larger than the experimental results. The results obtained with SCF theory are in good agreement with observed values. It shows that the SCF method is better than the Hückel method for the calculation of polarizability. The polarizability of the adenine-uracil pair estimated as the sum of the polarizabilities of adenine and uracil is compared with solving the eigenvalue problem for the A-U pair as an entity. The latter method gives considerably larger polarizability than the first method. Comparison of the calculated and experimental data for the two methods shows that the latter method is the superior when it comes to estimating the polarizability of the A-U pair. The transition moment of the A-U pair decreases in comparison with the sum of the transition moments of A and U. On this basis, the author has suggested that the delocalization of $\pi$-electron through the H-bonds may be part of reason for hypochromism.

In addition to the electronic properties of DNA presented in [124,125], one-dimensional models have been applied to calculate the energy bands of the delocalized electrons in DNA [126]. The single site potentials used for the calculations are (i) the $\delta$-function or (ii) the harmonic oscillator potential. The parameters of these potentials are defined by fitting the experimental value of $2.42 \mathrm{eV}$ for band gap. The authors studied the periodic diatomic chain of such potentials, and a disordered chain the quasi-periodic or virtual limit. The relevant gap for a pure A-T chain is $2.296 \mathrm{eV}$, for a pure G-C sequence the gap is $1.816 \mathrm{eV}$. Afterwards, the authors replace the disordered chain by an ordered one with each site carrying a potential which is the average of the different types of potentials. Thus, for the $\delta$-function chain this procedure gave an energy gap of 2.057 $\mathrm{eV}$, and for the harmonic oscillator case the energy gap is $2.056 \mathrm{eV}$. However, when considering the possibility of charge transport in DNA there are many contradictions within standard electron-transfer (ET) theory $[127,128,129]$.

Muon spin relaxation studies of DNA have revealed distinct differences in the behavior of double stranded DNA compared to single stranded DNA [130] and the free bases. For interpretation of the results $a b$ initio DFT calculations have been performed. Recently, the charge transfer (CT) and exciton states of stacked DNA base oligomers were studied by long-range corrected TD-DFT functionals for an improved determination of CT states among low lying electronic excitations of stacked DNA base oligomers [131]. The results suggest that the CT states of DNA oligomers are higher in energy than the bright exciton states.

\section{The pigments, cofactors and so on}

A detailed review of calculations of the electronic properties of pigments and cofactors by Hückel MO method was presented in [15], where the following themes were also considered: the principles of the MO method, the electronic structure of important biochemical substances, and the electronic aspects of enzyme reactions. In the Hückel method there exists some non-uniqueness (ambiguity) originating in the choice of the parameters of the method. However, the ideas presented by the authors of this book, especially the idea of the role delocalized $\pi$-electrons in the bioenergetical processes, remain important. The stereoelectronic properties of photosynthetic and related systems were investigated by many groups of scientists. They have used the different methods of quantum chemistry. So, Otten [132] and Nagae and Yomosa [133] fulfilled the Pariser-Parr-Pople $\pi$-electron SCF-MO CI studies of BChl a+. Knop and Stichtenoth [134] and Gouterman with colleagues [135] have presented Pariser-Parr-Pople calculations on chlorine; and Maggiora and Weimann 
Role of quantum chemical calculations in biophysics with a historical perspective

[136] fulfilled CNDO/S all valence electron studies. In work [137] the authors applied CNDO/S3. The most promoted method was used by Shipmann with colleagues $[138,139]$. In the work [138] ab initio CI calculations on the ground and lower excited singlet and triplet states of magnesium chlorine and chlorine were made. This work deals with the electronic characteristics and spectra of two reduced porphyrins, chlorine and magnesium chlorine. These molecules are important as simple analogs of chlorophyll systems, because the intense red absorption band of the chlorine is also an essential feature of the spectra of chlorophylls. The detailed characterization of many low-lying singlet and triplet states of chlorine and magnesium chlorine was compared to experimental data. The calculations included the configurational composition of each state and the corresponding transition energies, oscillator strengths, and electron density.

Such calculations were made for ethyl chlorophyllide $a$ (Et-Chl $a$ ) [140] and ethyl bacteriochlorophyllide $a$ (Et-BChl $a$ ) [141]. In [139] similar calculations were carried out for Et-Chl $a$ and Et-BChl $a$ cation radicals (Et$\mathrm{Chl} a^{+}$and Et-BChl $a^{+}$). These substances are very similar to chlorophyll $a(\mathrm{Chl} a$ ) and bacteriochlorophyll $a$ (Bchl $a$ ), which have essential biological meaning. The biochemical significance of Chl $a^{+}$and Bchl $a^{+}$is determined by the photosynthetic energy conversion in green plants and purple bacteria. The photo-oxidation of in vivo photosynthetic systems is apparent as a bleaching of the long wavelength absorption band attributed to $\mathrm{Chl} a$-containing or BChl $a$-containing reaction centers, and formation of oxidized systems which bring about EPR and optical spectra characteristic of $\pi$-cation radicals[142,143]. The observations from EPR and ENDOR studies maintained the idea of a bridged dimeric cation as the species formed after electron transfer in the photo-oxidation process [143].

The $a b$ initio configuration interaction method used in these works has been described in detail in papers $[144,145]$. Briefly, the configurations for given excited state are built from an orthonormal basis of molecular orbitals which are taken from self-consistent-field ground state wavefunctions. Details of the ground state $a b$ initio SCF calculations, which use a basis of floating spherical Gaussian orbitals (FSGO) obtained using the molecular fragment technique $[146,147]$ have been presented in works $[148,149]$. It is of interest to compare the results of Petke et al. [139] with those of Otten [132] and Nagae and Yomosa [133]. When comparing the computed optical spectra in [139] with the PPP results, a general qualitative agreement is seen in the description of absorption spectrum up to $20,000 \mathrm{~cm}^{-1}$ which is shown to consist of many low-intensity 'background' transitions. At the same time PPP calculations predict only a pair of transitions as contributing to the high-intensity region from $23,000 \mathrm{~cm}^{-1}$ to $28,000 \mathrm{~cm}^{-1}$; whereas the results of Petke et al. [139] shows four intense transitions in this region. This means that last work shows a larger total number of states over the full spectrum than is predicted from PPP results. It may well be due to both the extensive CI formulation applied here, and the treatment of all side chains and substituents. In work [150] the calculations of the electronic structure anion-radical and excited states of Ni-porphyrins were fulfilled by the Pariser-Parr-Pople $\pi$-electron SCF-MO CI method. For all cases the atom charges and bond orders were received. The calculated values were applied for the explanation of the optical properties of molecules under consideration.

Plato et al. carried out a molecular orbital investigation of dimer formations of bacteriochlorophyll $a$ [151]. The space structure of the primary electron donor in bacterial photosynthesis is very important because of their role in the charge separation in the reaction center. Norris et al. firstly proposed that the primary donor is a bacteriochlorophyll dimer. Deisenhofer with colleagues fulfilled an X-ray analysis of RC of Rhodopseudomonas viridis [48] and demonstrated the existence of a BChl dimmer. The theoretical study of one or several stable dimeric conformations of BChl might help to understand the function of the dimer on the primary photosynthetic events. The three stable dimer configurations of BChl $a$ were obtained by a selfconsistent-field molecular orbital calculations (all valence electrons, restricted Hartree-Fock, INDOparameterization, perturbational treatment of spin polarization). The method included geometry optimization by energy minimization. The results from magnetic resonance experiments were also compared with calculated spin-density distributions of the cation radicals: the largest energy of binding was received from a strong overlap of the $\pi$-systems together with the formation of two symmetrical $\mathrm{Mg}$ - $\mathrm{O}$ bonds between the monomeric components. This structure was very similar to the determined X-ray structure of the BChl $b$ dimer in Rps. viridis reaction center [48]. Possible perturbations of the dimer structure by the protein environment were discussed. 
Role of quantum chemical calculations in biophysics with a historical perspective

In [152] the calculated isotropic hyperfine coupling constants (B3LYP/EPR-II//BLYP/DZVP(D)) of the chlorophyll $a$ radical cation and anion were reported and are in good agreement with the experimental results, especially for the methyl groups. No larger differences in the hyperfine coupling constants (hfcs) between Chl $a^{+} \bullet$ and $\left(\mathrm{Chl} a^{\prime}\right)+\bullet$ were predicted to occur. At the same time the enol forms of Chl $a+\bullet$ gave rise to significantly altered hyperfine coupling constants in comparison with the respective keto form. In addition to the chlorophyll systems, the epimer and 01 enol of bacteriochlorophyll $a$ have also been calculated. From a comparison with experimental data it was concluded that the enol forms did not occur in photosynthetic reaction centers. On the bases of calculations under consideration an enolic form of Chl $a$ was therefore unlikely to contribute to P700.

In [153] DFT/B3LYP calculated structural parameters using the 6-31G(d) basis set showed agreement with the X-ray diffraction data for the structure of ethyl chlorophyllide (A). Normal vibration frequencies and absolute IR intensities of ethyl chlorophyllide (A) were also calculated. The role of saturated substituents in stabilization of radical ion states of chlorophyll was discussed. The effective force field of ethyl chlorophyllide (A) was obtained in independent and dependent internal coordinates. The IR vibrational spectrum was modeled and the experimental IR spectrum of chlorophyll (A) was interpreted on the basis of the calculation.

The study of the IR spectra and structures of all-trans- $\beta$-carotene and its 15,15 -cis isomer is carried out by Berezin et al. using the method of the previous paper [154] relative to the protective function of carotene in photosynthesis of higher plants. The effective harmonic force fields are received and the interpretation of the experimental infrared bands was given, and the $\beta$-ionone-ring vibrations were singled out. Using the structural similarity between natural caratenoids and stable radical molecules, they have put forward the hypothesis that the methyl groups of the в-carotene molecule play a protective, shielding role, that is, these groups are stabilizing biradical excited triplet state that arises in the process of triplet-triplet energy transfer.

Now we are turning to the quinones, which are known to play a major role in photosynthesis and respiration. Moreover, they have been detected in a large number of enzymes and proteins. The main function of quinones is to be mediators of the electron transport. Eriksson et al. have applied hybrid and gradientcorrected density functional methods to study theoretically the electronic and magnetic properties of neutral and charged quinine and plastoquinone radicals [155]. More precisely, the systems studied were 1,4benzoquinone ground-state singlet and first excited state triplet, the radical anion, the radical cation, and the singly and doubly protonated anion radical. H-bonded water molecules were taken into account in the studies of the radical anion, and influence of the hydrocarbon tail at the 3-position was studied for anion, cation, and protonated quinone. The H-bonded group is found to increase the electron affinity of the quinines by ca. 0.6 $\mathrm{eV}$. This is of crucial importance for the understanding of the photosynthetic processes. Further, the energetics of the electron-proton/H-atom transport in the quinone pool between photosystem 2 and 1 in higher plants are considered.

O'Malley studied spin density and hyperfine coupling for H-bonded 1,4-naphtosemiquinone and phyllosemiquinone anion radicals to clarify the influence of the H-bond on the above-mentioned characteristics [156]. The influence of H-bonding on the spin density distribution was shown to lead to a redistribution of $\mathrm{Z}$ spin density from the semiquinone carbonyl oxygens to the carbonyl carbon atoms. The effect of in plane and out of plane $\mathrm{H}$-bonding was studied. Out of plane H-bonding was shown to give rise to a significant delocalization of spin density on to the H-bond donor heavy atom. Good agreement is observed between calculated and experimental hyperfine couplings. Comparing of calculated hyperfine couplings with experimental determinations for the A1 phyllosemiquinone anion radical presented in Photosystem I (PS I) of higher plant photosynthesis indicated that in vivo radical may have a $\mathrm{H}$-bond to the $\mathrm{O} 4$ atom only as opposed to $\mathrm{H}$-bonds to each oxygen atom in alcohol solvents. The H-bonding situation appears to be the reverse of that observed for QA in the bacterial type II reaction centers where the strong $\mathrm{H}$-bond occurs to the quinone $\mathrm{O} 1$ oxygen atom. Perhaps for different types of reaction centre the presence or absence of the non-heme Fe(II) atom may well determine which type of H-bonding situation exists at the primary quinone site which may determine the direction of subsequent electron transfer.

Rinyu et al. [157] fulfilled the semi-empirical quantum chemical calculations (AM1 implemented in MOPAC93) for the role of the head group substituents in the redox functions of the quinones. In this work authors presented the ionization potential and the atomic charges by Mulliken population analysis of ubiquinone species 
Role of quantum chemical calculations in biophysics with a historical perspective

substituted with different isoprene subunits (2,3-methoxy-5-methyl-6-isoprenoyl-1,4-ubiquinone and 2,3,5,6tetramethyl-1,4-ubiquinone). The authors guess that the stabilization of light separated charges depends on the steric and/or electronic properties of quinones bound to the photosynthetic reaction center protein. The symmetric charge distribution of duroquinone is unfavorable for the point of view of the charge stabilization.

Effects of solvent on structural and electronic properties of spin label (4-amino-2.2.5,5 tetramethyl-3imidazoline-N-oxyl) was studied by Ikryannikova et al. [158]. Imidazoline-based nitroxide radicals are applied as spin probes for medium $\mathrm{pH}$ and polarity. These spin labels are used in studies bioenergetics processes. Practical application of spin probes is based on sensitivity of their EPR spectra to molecular mobility and properties of environment (polarity, $\mathrm{pH}$, microviscosity). Strictly speaking, the properties of these spin labels depend on protonation of radical and aqueous shell. The calculations were performed with density functional theory (DFT) using the Perdew, Berke, and Ernzerhof (PBE) exchange-correlation (XC) and the Becke-exchange and Lee, Yang and Parr-correlation (BLYP) XC functionals. These generalized gradient approximation (GGA) XC functionals are functions of the electron density gradient in addition to the electron density. The PBE XC functional is one of the best GGAs [159]. The BLYP GGA XC functional has been often used for calculations of spin density in nitroxide radicals. The results demonstrate that the spin probe protonation occurred at the nitrogen atom of the imidozoline ring rather than at the amino group. The authors demonstrated that the protonation of 4-amino-2.2.5, 5 tetramethyl-3-imidazoline- $\mathrm{N}$-oxyl results in a decrease in a spin density on the nitrogen atom of the nitroxide fragment. For study of spin-label hydration effects, the authors created a water shell around a spin-label molecule, gradually going from 2 to 41 water molecules surrounding the probe. The spin density on the nitrogen atom of the nitrogen atom of the nitroxide fragment increased with the extension of the water shell around spin probe. Protonation and hydration of spin-label molecule produced certain changes in bond lengths and valence angles. The main conclusion of this work is the following. The H-bonded cluster of more than 41 water molecules could be viewed as the model for simulation of spin probe hydration effects.

Quantum mechanical/molecular mechanical (QM/MM) calculations combining the DFT B3LYP/631G(d) and U-B3LYP/631G(d) XC functionals with the NAMD2 and AMBER4 force fields were used to study the electronic properties of one of the two domains of the plant blue-light receptors of the Phot family. These receptors mediate plant phototropism and contain two light, oxygen, and voltage (LOV)-sensitive domains as photoactive elements. The Phot-LOV1 domain from Chlamidomonas reinhardtii was studied [160]. The electronic properties and structural changes that follow blue-light absorption were studied by combined QM/MM simulations of the photocycle of the Phjt-LOV1 domain of C. reinhardtii [160]. This allowed one to resolve the structural changes and study the electronic processes accompanying flavin-cysteinyl adduct formation. The calculations allowed authors to study the pathway for flavin-cysteinyl adduct formation. This process proceeds via a neutral radical state provoked by hydrogen atom transfer from the reactive cysteine residue, Cys57, to the chromophore flavin mononucleotide. The adduct formation does not produce any larger scale conformational changes in the Phot-LOV1 domain.

Jalkanen et al. applied the modern quantum chemistry methods for the prediction of vibrational spectra and structures for biomolecules in the different surroundings [161,162,163,164,165,166,167]. They presented the structures, energies, vibrational frequencies, vibrational absorption (VA) intensities, vibrational circular dichroism (VCD) intensities and Raman scattering intensities for the conformers of small peptide Leuencephalin (LeuE). This peptide is biologically active and found endogenously in many animals and humans [161]. The author used the hybrid Becke 3LYP exchange-correlation potential and the 6-31G* basis set.

The VA and VCD spectra simulated by Abdali et al. have been compared to the measured VA and VCD spectra of LeuE in DMSO [168]. On this basis the structure of LeuE has been proposed. Only the non-ionic neutral species of LeuE was taken into account, as this is the species thought to be present in the nonpolar membrane environment where LeuE binds. The crystal structure of LeuE bound to its receptor has not yet solved. The question of the species and conformation in solution versus bound to the receptor is still being investigated, as LeuE is thought to bind via an induced fit, versus the lock-and-key mechanism. In doing so, it has been shown that many receptor proteins also more rigid on binding, and it may actually be easier to get a crystal structure 
Role of quantum chemical calculations in biophysics with a historical perspective

for the ligand bound receptor than it is to get either the crystal structure for either the free ligand or free receptor.

\section{QUANTUM CHEMISTRY STUDIES THE BIOENERGETIC PROCESSES.}

\section{A. The generation and transfer of the charges states in photosynthesis.}

After Deisenhofer et al. determined the structure of the bacterial photosynthetic reaction center [48], Kuhn considered the mechanism for the electron transport in the reaction center [169]. The rate constant of the electron transport between the different carriers was calculated by Fermi golden rule; $K=(2 \pi / \hbar) \varepsilon^{2} S$. In the formula the electron perturbation energy $(\varepsilon)$ is the matrix element of perturbation energy on the electron wave functions of donor and acceptor before and after the electron transfer. The value $S$ is the Frank-Condon factor. In this work the electron transfer rates were determined for all stages of the electron transport in the reaction center. In doing so, the protein part of reaction center was taken into account as a rigid dielectric continuum, so that the free energy loss by solvent reorganization can be neglected. The electron wave functions in the corresponding matrix elements were chosen as the functions given by the free electron model. The rate constant for different stages of the electron transport in reaction center were in good agreement with the experimental data.

In work [170], called "Theoretical investigation of the rates of electron transfer processes $\mathrm{Q}^{-}{ }_{1}+\mathrm{Q}_{2} \rightarrow$ $\mathrm{Q}_{1}+\mathrm{Q}_{2}$ and $\mathrm{Q}_{1}^{-}+\mathrm{Q}_{2} \rightarrow \mathrm{Q}_{1}+\mathrm{Q}^{2-{ }_{2}}$ in photosynthesis", the characteristics of the quinones were estimated to study the influence of surroundings on the rate of the electron transport between $\mathrm{Q}_{1}$ and $\mathrm{Q}_{2}$. The study was carried out by using the Marcus theory of long-range electron transport in solution [57]. The molecular reorganizational parameter $\lambda$, the free energy change $\Delta \mathrm{G}^{0}$ for the overall reaction, the electronic matrix element $\mathrm{H}_{\mathrm{AD}}$ were calculated from the INDO-optimized geometries of molecules $\mathrm{Q}_{1}, \mathrm{Q}_{2}$ and histidine. The study has given the standard reduction potential of the process $\mathrm{Q}_{1}^{-}+\mathrm{Q}_{2} \rightarrow \mathrm{Q}_{1}+\mathrm{Q}_{2}$ as $127 \mathrm{mV}$ and the midpoint potential of $\mathrm{Q}_{1^{-}}+\mathrm{Q}_{2}\left(\mathrm{H}^{+}\right)+\mathrm{H}^{+} \rightarrow \mathrm{Q}_{1}+\mathrm{Q}_{2} \mathrm{H}_{2}$ as $174 \mathrm{mV}$ at $300 \mathrm{~K}$ at $\mathrm{pH}$ 7. The experimental value is $130 \mathrm{mV}$ for the first process; for the second process, the midpoint potential is not precisely determined. Both the transfer processes took place in the absence of $\mathrm{Fe}^{2+}$ ion. The main role of $\mathrm{Fe}^{2+}$ ion appears to be the support of the rigidity of the matrix by holding the two histidine molecules firmly in place. The authors deemed that the Marcus theory of long-range electron transfer can be successfully applied to the study of electron transfers in the semirigid condensed phase that is located inside the thylakoids.

The quantum chemistry with the semi-empirical methods and the ab initio ones turned out to be very useful for understanding of absorption spectra of the photosynthetic pigments and the electronic properties of primary donors and acceptors. The extensive review of results obtained by 1980's is listed in book [171].

Now we consider the works in which peculiarities of electron transfer in primary processes of photosynthesis were studied. The results received by X-ray analysis of crystals of the reaction centers of the purple bacteria showed [48] that the pigment and cofactors (bacteriochlorophyll of the special pairs, monomeric bacteriochlorophylls, monomers of bacteriopheophytin and two quinones) are located symmetrically along L and M subunits of protein complex RC. However, in the kinetic experiments it was shown that the electron transfer along L subunit occurred about five times quicker than along $M$ subunit [172]. Why the electron chooses one of two similar paths is not clear? The study of the structure of the RC protein complex in detail showed the essential asymmetry in the location of the polar amino acid residues in $\mathrm{L}$ and $\mathrm{M}$ chains of complex. That's why the assumption appeared that such asymmetry can influence the electronic properties and energetic characteristics of the nearest pigments in L and M chains of RC. The authors [173] explained the advantage of $L$ chain by the protonation of glutamate near bacteriochlorophyll of special pair of Ll chain; in that place in $\mathrm{M}$ chain the nonpolar amino acid valine being located. The calculations were carried out in model of electrostatic interactions of pigment molecules with the RC polar surroundings; the influence of this asymmetry on the electronic and energetic characteristics of special pair on which the 
Role of quantum chemical calculations in biophysics with a historical perspective

charge transfer begins was not studied. This problem was investigated in work [174] by quantum chemistry methods. The authors treated the influence of nearest to the special pair polar amino acids. The calculations were fulfilled in the scope of the electrostatic interaction between the $\pi$-electron system of dimer of special pair and the electrical charges on the amino acids of RC proteins. At first the authors have determined the one-electron wave function (molecular orbitals) and corresponding one-electron energy for $\pi$-system of bacteriochlorophyll molecule. The Roothaan method in NDO approximation is applied. The integrals of electrostatic interactions were estimated by Mataga method [175]. The determinant of Roothaan equations was factorized by group orbital basis, which are the basis of irreducible representations of group $\mathrm{D}_{2 \mathrm{~h}}$. The molecular orbitals obtained are satisfactory, see data listed in [176]. After that the authors took into account the electrostatic influence of nearest polar amino acids of RC on the electronic characteristics of the special pair. They considered the following amino acids: histidine (L 168), histidine (L173), threonine (L248), histidine (M200), tyrosine (M195). The specific arrangements of these amino acids are listed in work $[48,177]$. The operator of electrostatic interaction of polar amino acids and $\pi$-system of dimer is considered as the small perturbation in Hartree-Fock equations.

To solve this problem, the authors [174] used first order perturbation theory because the expectation value of the perturbation operator is less than the eigenvalue difference $\left|\varepsilon_{\mathrm{i}}-\varepsilon_{\mathrm{k}}\right|$. The resulting eigenvalue differences were: $\Delta \varepsilon_{9 \mathrm{~L}}=-0.2 \mathrm{eV} ; \Delta \varepsilon_{9 \mathrm{M}}=0.14 \mathrm{eV} ; \Delta \varepsilon_{10 \mathrm{~L}}=-0.16 \mathrm{eV} ; \Delta \varepsilon_{10 \mathrm{M}}=0.12 \mathrm{eV}$. The energies $\varepsilon_{9}$ and $\varepsilon_{10}$ for the special pair were determined using the one-configuration approximation, the ninth molecular orbital $\psi_{9}$ being the highest occupied orbital and the tenth one being lowest unoccupied orbital. The ionization potential of $\mathrm{BCH}_{\mathrm{MP}}$ for the first singlet excited state is $0.28 \mathrm{eV}$ smaller than the ionization potential of $\mathrm{BCH}_{\mathrm{LP}}$. The estimation the rate of electron transfer by the Jortner formula [178] shows that the ratio of the rate constants for $\mathrm{BCH}_{\mathrm{MP}}$ and $\mathrm{BCH}_{\mathrm{LP}}$ is about ten. These results seem to be strange, because for $\mathrm{BCH}_{\mathrm{MP}}$ the electron is transferred via the L-chain. However on the closer inspection of structure of RC it can see that the difference of distance between $\mathrm{BCH}_{\mathrm{MP}}$ and $\mathrm{BCH}_{\mathrm{LP}}$ on one hand and the primary acceptor $\mathrm{BPH}_{\mathrm{L}}$ is not so large (near $0.1 \mathrm{~nm}$ ) as compared with the average distance between the electron carriers. Perhaps the influence of charge of the nearest polar amino acids is more essential than the distance difference.

The electronic structure of the RC chlorophyll dimer was studied using the ab initio molecular orbital scheme and pseudocharges and dipoles method which takes into account the electrostatic effect of the surrounding proteins, pigments, and aqueous solvent [179]. Let us briefly review the structure of RC from the photosynthetic bacteria Rhodopseodomonas viridis. It consists of four protein subunits: L (light), M (medium), H (heavy) and cytochrome. The RC protein part surrounds the pigments: four bacteriochlorophyll (BChls), two bacteriopheophytins, one menaquinone, one nonheme $\mathrm{Fe}$ and one ubiquinone. The pigments make up two subunits, L and M that are almost symmetrical. The two BChls, named BClp and BCmp, belonging to $\mathrm{L}$ and M-subunits correspondingly, is usually called the "special pair $\mathrm{P}$ ". It is well-known that the electron transfer processes proceeds only along L-subunit. The cause of it is not clear. The total energies of $\mathrm{BClp}, \mathrm{BCmp}$, and $\mathrm{P}$, the differences from the energies when taking the electrostatic effect into account, and its dimerization energy were determined. The ionization potentials of above-mentioned components in gas phase and solvated system were determined. The main conclusions of the article are the following. First, the electron is removed slightly more easily in vacuum from $\mathrm{BCmp}$ than from BClp. This is possible since the molecular plane of $\mathrm{BCmp}$ is clearly distorted, but not so distinctly in BClp. Therefore, the ionization from $\mathrm{BCmp}$ occurs more easily than from BClp. Perhaps, this smaller ionization potential is determined partially by the chlorophyll molecular structures themselves, as this happens even when the electrostatic potentials of the surroundings is ignored. The tendency is for the ionization potential of $\mathrm{BCmp}$ to be enhanced by the addition of the electrostatic potentials creating the polarizable environment. The difference in the ionization potentials of $\mathrm{BCmp}$ and $\mathrm{BClp}$ is large as $0.6 \mathrm{eV}$. The authors guessed that for the correct treatment of problem under consideration the induced dipole moments should be included in the effective Hamiltonian for the $a b$ initio MO method.

The electron transfer between the primary and the secondary quinone of the bacterial RC has been investigated theoretically [180]. It was supposed that in this system that a mobile electron which is injected on one end of the chain can be transported to the opposite end by step-by-step switching the positions of the $\mathrm{H}$-bonded hydrogens. In doing so, not only protons but also hydrogen atoms are involved. In such manner the 
Role of quantum chemical calculations in biophysics with a historical perspective

formation of an $\mathrm{H}$-bonded chain linking the two redox centers can play the essential role in the mechanism of electron transfer. This type of the electron transport was called proton assisted electron transport (PAET). So PAET mechanism was considered in the next system: $Q_{A^{-}} \ldots H-H i s-F e-H i s-H \ldots Q_{B}$.

The authors have replaced $\mathrm{Fe}^{2+}$ by $\mathrm{Zn}^{2+}$ to simplify computations. The energetic calculations have been received at $a b$ initio self-consistent level of theory using GAMESS package and the standard 6-31G basis set, expanded with polarization functions on all the atoms participated in H-bonds. The calculations of energy predicted that the various stages of the PAET mechanism were all slightly exegetic $(6.1 \mathrm{kcal} / \mathrm{mol})$. The potential energy profile was proposed to describe the proton vibrations around two sites $\mathrm{Q}^{-}$...H - His and $\mathrm{Q}^{-}$ H... -His. The authors have calculated the vibrational frequencies associated with proton oscillation, solving the Schrödinger equation variationally. The calculated frequency for the proton oscillation is $2656 \mathrm{~cm}^{-1}$, shifting to $2016 \mathrm{~cm}^{-1}$ on deuteration. This result permits to verify calculations.

In last time the quantum chemistry methods were applied to nanotechnology. In work [181] the authors studied the atomic-scale coupling of photon to single-molecule junctions containing biologically important component magnesium porphine (MgP). The chlorophyll, the main component of the photosynthetic systems, is a derivative of the MgP. The authors of this work showed the coupling of photons to a single molecule of MgP in a double-barrier junction of the scanning tunneling microscope (STM) through a two-step process of photo-induced resonant tunneling. In this process an electron is photo excited to a higher level in the tip and then tunnels resonantly to a molecular state of the MgP. For the interpretation of the experimental data the DFT calculations on the planar MgP molecule were fulfilled by using HyperChem 7.0. The energy separation between the lowest unoccupied molecular orbital (LUMO) and the LUMO +1 is $\sim 1.6 \mathrm{eV}$. This difference corresponds to $800-\mathrm{nm}$ light observed in the tunneling electron-induced fluorescence spectra.

The time changes of pigment-pigment and pigment-protein interactions in antenna light-harvesting complexes of bacteria were investigated with method that combines molecular dynamics simulations with quantum chemistry calculations and polaron model analysis [182]. These simulations were done on an 87055 atom system, contained a LH-II complex of Rh. molischianum build-in in a lipid bilayer and surrounded with appropriate water layers. The authors fulfilled $400 \mathrm{ab}$ initio quantum chemistry calculations for each of the 16 B650 bacteriochlorophylls. The geometry of this system was received from the preliminary molecular dynamical simulations. As a result of the approach, the fluctuations of pigment excitation energies as a function of time were determined. On the basis of these results the authors introduced a time-dependent Hamiltonian for the B850 exciton system determining the absorption spectrum inside linear response theory. Furthermore, a polaron model is applied to study both the excitonic and coupled photon degrees of freedom by quantum mechanics. The constant of the exciton-phonon coupling and the phonon spectral function for polaron model are received from the molecular dynamics and quantum chemistry calculations. The proposed approach permits to determine that excitons in the B850 BChl ring under consideration are spread over five pigments at room temperature. Moreover the model gave possibility to calculate the absorption and circular dichroism spectra of the B850 excitons.

\section{B. The proton transport in photosynthesis.}

The intensive area of application of quantum chemical approaches is the coupled electron and proton transport in bacterial and plant photosynthesis, namely: early coupling events in the vicinity of the secondary quinone. The one of problem is what molecular properties of the system account for its ability to synchronize electron transfer during $\mathrm{Q}_{\mathrm{B}}$ reduction with proton uptake from the outer side of the membrane in chromophores of bacteria or thylakoids of higher plants [183]. Earlier, Tomchuk et al. studied theoretically the process of proton movement through $\mathrm{F}_{0}$ subunit of ATP synthase [184], using the polaron theory. The kinetic characteristics of the process, such as current density, polaron lifetime, and proton mobility, were estimated. In our study [185], we have surmised that electron transfer from histidine L190 to the secondary quinone $\mathrm{Q}_{\mathrm{B}}$ is followed by proton transfer. The hypothesis was tested in that study by calculating the energy of the quinone-histidine system. The semi-empirical MNDO (Modified Neglect of Diatomic Overlap) method 
Role of quantum chemical calculations in biophysics with a historical perspective

[186] was used as the optimal one in terms of accuracy required for solving our problem versus the computing speed. The calculations were done for various positions of the proton between quinone and histidine for various redox states of $\mathrm{Q}_{\text {В }}$ (neutral, singly reduced, and fully reduced). Inspection of the shapes of the curves obtained allowed some qualitative conclusions to be drawn. Namely, when the quinone is in the singly reduced state, an H-bond is likely to form; the bond between the proton and the doubly reduced quinone is covalent and its formation changes the distance between $\mathrm{Q}_{B}$ and histidine. In the work [185], we assessed quantitatively the position of the proton between the histidine and $\mathrm{Q}_{\mathrm{B}}$. Specifically, the probability density function of proton positions and the respective energy levels of the proton were determined by numerically solving the Schrödinger equation for the hydrogen of histidine in the histidine- $\mathrm{Q}_{\text {В }}$ system. The main results are the following. In computations, we varied the redox state of plastoquinone $\mathrm{Q}_{\mathrm{B}}$ (oxidized, singly reduced, and doubly reduced) and the distance between the nitrogen of histidine and the oxygen of $\mathrm{Q}_{\mathrm{B}}$. The calculated square moduli of the wave functions (which determine the probability density for the proton position along the line connecting the oxygen of quinone and the nitrogen of histidine) and the energy levels for the lowest three states of the proton are shown in $[183,185]$.

The single maximum of the ground-state wave function of oxidized plastoquinone is $1 \AA$ away from the nitrogen of histidine. The maxima of the wave functions corresponding to the excited states are not shifted farther than $0.5 \AA$ from this position. The lowest wave function of singly reduced plastoquinone $\mathrm{Q}_{\mathrm{B}}{ }^{-}$ has a broad maximum midway between the nitrogen of histidine and the oxygen of plastoquinone. The maxima of the wave functions corresponding to the excited states are far separated, suggesting H-bonding to the oxygen of $\mathrm{Q}_{\mathrm{B}}$. The single maximum of the ground-state wave function of doubly reduced plastoquinone $\mathrm{Q}_{\mathrm{B}}{ }^{2-}$ is $0.9 \AA$ away from the oxygen of $\mathrm{Q}_{\mathrm{B}}$. Sequential reduction of $\mathrm{Q}_{\mathrm{B}}$ shifts the maximum of proton position probability density in the direction from the nitrogen of histidine to the oxygen of $\mathrm{Q}_{\mathrm{B}}$. The resembling result was received in work [187] by the DFT/B3LYP/6-31(d) method.

So, the authors of the work [183] deemed that the same scheme of coupling of electron and proton transport may well take place in the cyt $b_{6} f$ complex and oxygen-evolving systems in chloroplasts. Some result in support of this suggestion was obtained in paper [188], in which the results of quantum chemical calculations of the proton position in the tyrosine $Y_{Z}$ - His D1-190 in the water-oxidizing complex of higher plants were presented. The main result was the following. When the system under consideration was in the neutral state, the proton is located $1 \AA$ away from oxygen atom of the $Y_{\mathrm{z}}$. After the $Y_{\mathrm{z}}$ is oxidized, the proton is shifted $1.7 \AA ̊$ farther, to a distance of about 1 Å from the nitrogen atom of His D1-190.

In work [189] the authors calculated the energies of complexes $\mathrm{P}^{+} \mathrm{Q}_{\mathrm{A}}^{-}$and $\mathrm{PQ}_{\mathrm{A}}$ in reaction center Rhodobacter sphaeroides taking into account their specific surrounding. The calculations were fulfilled for the different stable positions of protons. The optimization of structure and calculations of energy were made by semi-empirical PM3 method and DFT method (basic 6-31G*, functional B3LYP). The authors demonstrated the essential influence of proton positions on energy of transition $\mathrm{P}^{+} \mathrm{Q}_{\mathrm{A}}^{-} \rightarrow \mathrm{PQ}_{\mathrm{A}}$.

\section{The ion channels and the other structures.}

The ab initio calculations (RHF + MP2 (FULL), basis 6-31 $\mathrm{G}^{* *}$ ) were used for the study the proton migration in tyrosine stack under the action of $\mathrm{Mg}^{2+}$ ions and the electrical field. This system was a model of the proton channel in tubuline [190]. In this system there is only the energetic barrier of $0.72 \mathrm{kcal} \mathrm{mole}^{-1}$, easily surmounting by thermal movement.

The quantum chemical methods intensively were applied in the approaching the gating problem in functioning of ion channel [191]. The results of Gaussian 98 calculations (DFT method, B3LYP functional, 6$311 \mathrm{G}^{++}$basis) supported the hypothesis about the gating of bacterial ion channel. The authors of work have studied the role of proton in gating of potassium channel [192]. The density functional calculations were carried out, using Gaussian 98. The system under consideration included: one pair of guanidinium groups, the side chains of arginines responsible for carrying the proton current, a mobile proton and one, two or three water molecules. Several guanidinium spacings have been tried, all in the range of carbon-carbon 
Role of quantum chemical calculations in biophysics with a historical perspective

distances from 4 to $6 \AA$. The potential energy surface was received for each distance, and minimum path was founded for the proton at B3LYP/6-311G** level. It was found that under some conditions the proton could follow a path between guanidiniums that had no barriers greater than a few kT.

In work [193] the authors studied the quantum dynamics of the femtosecond photoizomerization of retinal in bacteriorhodopsin. At absorption of light, all-trans-retinal is tested a subpicosecond all-trans $\rightarrow 13-$ cis phototransformation involving torsion around a double bond. The reaction product initiates events in bacteriorhodopsin that cause pumping of a proton through protein. Quantum-chemical studies suggest that three coupled electronic states (the ground state and two closely lying excited states) participate in the motion along the torsional reaction coordinate. The evolution of the protein-retinal system under consideration on these three electronic surfaces has been calculated applying the full multiple spawning (FMS) method for non-adiabatic dynamics. With this method the time evolution of the population on the one of the product state, the 13-cis state, was investigated. The authors found that almost most of the population transfer occurs on a timescale of $300 \mathrm{fs}$, some population transfer continues during a longer timescale, extending beyond 1 ps. This paper gives us a framework for future studies of the photodynamics of retinal in bacteriorhodopsin and of other biological photo processes.

In paper [194] the authors studied the role of an H-bonded network in energy storage the lightdriven proton pump in bacteriorhodopsin.

This problem is stated as follows: how bacteriorhodopsin accumulates the light energy absorbed by the chromophore. In this work the authors fulfilled an ab initio quantum mechanical/molecular mechanical (QM/MM) vibrational normal-mode analysis of the binding place for the proposed $\mathrm{K}$ intermediate state, which generates 3 ps after photoabsorption and can be trapped at low temperature. A QM/MM routine [195] using the AMBER field were applied.

The analysis showed that the observed spectral changes mean that H-bonds among water molecule 402, Asp85 and the Schiff base weaken. This observation is essential to understanding the energy storage in bacteriorhodopsin.

The group of scientists $[196,197]$ undertook the attempt to apply the modern methods of quantum chemistry for modeling the catalytic mechanisms of guanosine triphosphate (GTP) hydrolysis in the different guanosine triphosphate-binding proteins (so called G-proteins). The authors used ab initio QM/MM simulations. The program package of this QM/MM method was based on two programs: PC GAMESS [198] and program TINKER (URL http://dasher.wustl.edu/tincer).

It was shown that the GTP hydrolysis reaction for the different G-proteins proceeds in uniform fashion. The following sequence of reactions occurs. At the first stage the $\gamma$-phosphate group is removed from GTP. After that the reaction water molecule carries out a nucleophilic attack on the phosphorus atom of the split phosphate group. At last the proton transfer and phosphate production take place. In doing so, a number of the stages in the hydrolytic reactions depend on the affinity of the catalytic amino acid for proton.

Using multiconfigurational quasidegenerate perturbation theory of the second order (the original technique aug-MCQDPT2) the authors of works [199,200,201] received assessing for the position of absorption maximum of retinal conformers, chromophores of green fluorescence protein (GFP), and flared fluorescence protein (asFP5950) in different protonated states. The aug-MCQDPT2 method together with QM/MM technique provides the means of calculating the energies of the vertical electron transitions for the chromophores in condensed matter. The approach gives one a possibility to study the influence of medium on the optical properties of the chromophore group in proteins.

Of fundamental importance for understanding the mechanism of proton transport is the role of long-range coupled motion in an enzyme catalyzed reactions [202]. In the work the authors presented a description of an enzyme-catalyzed reaction dominated by proton tunneling. They studied the reaction pathway for tryptamine oxidation by aromatic amine dehydrogenase (AADH). AADH is a tryptophan tryptophyl quinone-dependent quinoprotein. It catalyses $\mathrm{H}$ transfer by quantum mechanical tunneling. 
Role of quantum chemical calculations in biophysics with a historical perspective

Combining experiment determining the crystal structures of key intermediates and computer simulation with use of a combined QM/MM potential the authors received the following. It happened to be that data of the analysis of tunneling in AADH by computational methods is agreement with the experimental kinetic data received by using sopped-flow methods. Moreover the proton transfer occurs by tunneling over $\sim 0.6 \AA$. In this system tunneling is assisted by a short-range motion and no long-range coupled motion is needed. That's why the role of long-range coupled motions in promoted proton tunneling remains unsolved.

In [203] the structure and dynamics of the Fe-CO bond in the oxymyoglobin active site and its cobaltsubstituted analog were studied. The author used the DFT, CMPD (Car-Parrinello molecular dynamics) $[204,205]$ and hybrid QM/MM approaches. The results of calculations testify that both in the gas phase and in the protein the Fe-CO bond is linear and that's why the hypothesis that the $\mathrm{CO}$ in $\mathrm{MbCO}$ is hindered is not true. The protein discrimination for $\mathrm{Fe}-\mathrm{CO}$ bond is due to the $\mathrm{H}$-bonding between the $\mathrm{O}_{2}$ ligand and the His64 residue. It should be noted that the CMPD method was broadly applied in the investigations of the biological systems [203].

\section{The oxygen evolving center (OEC) in plants}

The developed model for the water splitting system of thylakoid membranes using the molecular orbital study system was presented in [206]. The authors of this work have made the molecular orbital calculations of the process of water oxidation. The ab initio LCAO (linear combination of atomic orbital) MO UHF (unrestricted Hartree-Fock calculations) were used. It was shown that some valence states of manganese work as catalytic metal cations, which decompose $\mathrm{H}_{2} \mathrm{O}$ into $\mathrm{H}^{+}$and $\mathrm{OH}^{-}$(or $\mathrm{OH}$ radical). The potential energy curve for the H-bond between two water molecules was obtained for the different states of Mn ion located near water dimer. The authors proposed that the system under study must have a protonaccepting center which builds up an H-bond with a water molecule to be oxidized by the manganese. At later time this supposition was confirmed in the structural findings [207,208,209].

The quantitative model of water splitting system using the quantum chemical consideration was presented in [210]. It described the geometry of the $\mathrm{O}_{2}$ evolving complex and energetics of the process of water splitting. Two molecules of water are bound to four amino acid residues (histidines) by H-bonding in the active site, and this aids in the $\mathrm{H}_{2} \mathrm{O}$ splitting by pulling proton through the H-bond. Four Mn atoms are divided into two groups: one group is in the active site and helps in the water splitting and other group is in the interface between the Mn-containing intrinsic protein and extrinsic proteins (14-18, 23-25, 32-34 kD), acting as an electron carrier between the $\mathrm{O}_{2}$ evolving complex and P680. However, the modern structural data $[207,208,209]$ were not taken into account in this work.

The new structural data were used in [211]. In author's opinion, the problem of oxygen formation in photosynthesis is the following. It is not clear how it is possible to remove four protons and four electrons from two water molecules upon absorption of four quanta and while avoiding the formation of reactive oxygen species $\left(\mathrm{O}_{2}^{-}, \mathrm{H}_{2} \mathrm{O}_{2}, \mathrm{OH}\right)$, and to produce a stable molecule of oxygen. The water-oxidizing complex is so efficient that does not need help from additional defense systems such as antioxidants, superoxide dismutases, and catalases. In this work the new model of the oxygen evolving complex (OEC) based on the cubane-like $\mathrm{Mn}_{3} \mathrm{CaO}_{4}$ model [206] was proposed. In doing so, the authors used the ligand field theory and the rules of 18 and 10 electrons for stable shells of $\mathrm{Mn}$ and $\mathrm{Ca}$ ions respectively. By the same procedure they added model [206] by chlorine and water molecules. The authors proposed the mechanism of water oxidation in which calcium ion and remote manganese Mn4 play the main role. The scheme of the redox state transitions between discrete S-states of OEC at excitation by short pulse of light were supposed. This model states that using manganeses as necessary element of OEC is determined by their transitional character. The presence of calcium in OEC is explained by its stability in state with completely filled shells and little affinity for electron in this state. The cubane-like cluster does not allow Mn4 to fill their molecular shells by electrons from nearby oxygen that makes Mn4 to oxidize the molecule of water. In this work the estimation of the system energy in S-states was carried out. The authors used the software package HyperChem and semiempirical method ZINDO. To simplify the calculations, they used $\mathrm{HCOO}^{-}$groups instead of aspartic and 
Role of quantum chemical calculations in biophysics with a historical perspective

glutamic acids, and the $\mathrm{NH}_{4}{ }^{+}$group instead of histidine. To optimize the system the authors changed the length of the bonds with those molecules that were not obtained from the structure [206], i.e., four water molecules and the chlorine ion. In doing so, it was obtained that the bond energy in each of S-states is negative. It means that this configuration of system under consideration is stable.

M. Kusunoki presented the interesting new mono-manganese mechanism of the photosystem II oxygen evolving reaction catalyzed by a unique $\mathrm{Mn}_{4} \mathrm{Ca}$ cluster [212,]. This model is assumed to be composed of $\mathrm{Y}_{\mathrm{z}}$ (Y161), Gln165, Asp170, Glu189, His 190, His332, Glu333, His337, Asp342, and Ala344 from D1 protein, Glu354, and Arg357 from CP43 protein, a negative $\mathrm{Cl}$ - ion suggested to be bridging between positive Arg357+ and $\mathrm{Ca}^{2+}$ ion, and a monomer-trimer type $\mathrm{Mn}_{4} \mathrm{Ca}$ to be proposed. DFT/B3LYP computations have shown that the proposed structure is consistent with the observed EPR, EXAFS and isotope ${ }^{18} 0$ exchange kinetics data in a Kok's $\mathrm{S}_{\mathrm{i}}$-state cycle.

The structural models of the oxygen-evolving complex of photosystem II (PSII) in the dark-stable $\mathrm{S}_{1}$ state, as well as in the reduced $S_{0}$ and oxidized $S_{2}$ states, were considered in work [213]. In doing so, acid residues, water, hydroxide, and chloride ion have been taken into account. The authors applied the modern quantum mechanics/molecular mechanics (QM/MM) hybrid methods for the description of the structural and electronic properties of the system under consideration. The proposed models were based on the X-ray diffraction structure of cyanobacterial PSII, which was made at $3.5 \AA$ fesolution (PDB access code 1S5L). The authors remarked that the proposed X-ray diffraction models remain rather controversial because of their small accuracy in the definition of the distances between atoms. The questions asked in this work are the following. What are sizes of vectors between the different Mn atoms? Where is chloride ion located? What are the positions of water molecules? The main results of this work are the following. For $S_{1}, S_{0}$, and $S_{2}$ states of system under study the structural and electronic characteristics were determined including the distances between atoms and bond angles. For this models the simulation of the extended X-ray absorption fine structure (EXAFS) spectra were carried out. The authors considered that the proposed QM/MM models are agreed with X-ray diffraction models and EXAFS spectrum data. The proposed place of calcium-bound chlorine ligand is consistent with pulsed electron paramagnetic resonance data, received from acetatesubstituted PSII. In that way this model is of essential interest for scientists working in this field.

In another work it was shown that the generally accepted QM/MM models have coincidence with the Xray model [209], the differences being in the protonation states, in the number of ligated water molecules, or in the coordination of labile ligands which exist. Only two spin states of Mn were found for the $S_{1}$ base state. This is so called model A with $\operatorname{Mn}(1)=I V, M n(2)=I V, M n(3)=I I I, M n(4)=I I I$, in which the dangling manganese is pentacoordinated. In model B with $\mathrm{Mn}(1)=\mathrm{IV}, \mathrm{Mn}(2)=\mathrm{III}, \mathrm{Mn}(3)=\mathrm{III}, \mathrm{Mn}(4)=\mathrm{IV}$, the dangling manganese is hexacoordinated and has an additional ligated water. The essential result obtained by the authors was that the cubane-like model of the inorganic core of the OEC of PCII, ligated with water, $\mathrm{OH}^{-}$, and $\mathrm{Cl}^{-}$and protein ligands, was a stable molecular structure even in the absence of the surrounding protein environment. In that way this model is of essential interest for scientists working in this field.

\section{Discussion, conclusions and perspectives}

Some words about application of the modern methods of quantum chemistry in biophysics. One of the most used spectroscopic techniques is nuclear magnetic resonance (NMR). Recently the prediction of NMR shielding via $a b$ initio and DFT methods has undergone a revival. Originally HF method were used to predict NMR shielding and gauge invariant atomic orbitals (GIAOs) were developed. It has been recently stated that the B3LYP method should not be used for the calculation of the electron spin resonance hyperfine structure (HFC) constants, nuclear resonance spin-spin coupling constants, magnetic shieldings and other properties [214]. Many groups continue to use the B3LYP method as it became the so-called Gold Standard for many DFT calculations, geometry optimizations (where dispersion forces and van der Waals interactions) are not important, frequency calculations and vibrational absorption (VA) or infrared (IR) intensities [215]. 
Role of quantum chemical calculations in biophysics with a historical perspective

But for magnetic properties, both ground and excited states, the B3LYP is NO LONGER the Gold Standard. The B3PW91 hybrid XC functional has been shown to be much better [214].

The calculation of the potential energy hypersurfaces is a much more difficult task for exited states as compared with the ground states. It is agreed that now the best method of calculation is the timedependent density functional theory (TDDFT) [216] approach, applying for excited-state calculations. However this method gives the wrong results for some states low-lying $\pi \pi^{*}$ states $[108,217]$.

It is interesting to compare method of multiconfigurational self-consistent field (MCSCF) and best density functional theory (DFT) based methods. Now it is deemed that the first more adequately describes the experimental data. However it is not surprising because DFT method contains more parameters in the basis functions and in the functional. At the same time the MCSCF method contains the free parameters only in the basis functions. The advantages and shortcomings of the different methods were discussed in work $[218,219,220]$. As a rule, the biological objects are the systems containing a lot of electrons and nuclei. That's why the choice of the quantum chemistry method of study is determined by the taste of author namely the aim of investigation, the computer capabilities and so on.

In conclusion, let us remark the main stages of penetration of quantum chemistry methods in biology. At first, they were applied for explanation of the optical and electronic properties of relatively simple and afterwards complex component of living organisms. After that the scientists began to study the structure and the electronic characteristics of biopolymers, proteins and nucleic acids, and the large protein-lipid complexes for which were received X-ray diffraction structural models. Among these were the reaction centers of the photosynthetic bacteria and higher plants, the ion channels, the mitochondrial respiratory complexes. Approximately at the same time the quantum chemistry methods were used in studying the main biological processes such as the electron and proton transports in photosynthesis and respiration, the gating in ion channels, and visual reception.

Some other aspects of quantum chemistry applications in biophysics are discussed in articles [221,222]. In addition to electronic circular dichroism, vibrational circular dichroism has been developed to the point where it can now be routine used in not only organic chemistry [223], but also in the field of molecular biophysics [224]. Indeed in the group of Professor Sandor Suhai, to whom this special focus issue of TCA is dedicated, many of the pioneering works on determining the causes responsible for the large changes VA and VCD spectra of amino acid and peptide in aqueous solution versus those of amino acids and peptides in the gas phase or isolated in non polar solvents like carbon tetrachloride, carbon disulfide and chloroform and chloroform- $\mathrm{D}_{3}$ have been undertaken on the alanine dipeptide, N-acetyl L-alanine N'methylamide $[225,226,227,228,229,230]$, L-alanine [163,224,227,230,231,232,233], L-alanyl L-alanine $[224,227,234,235]$, L-histidine [236], tri-L-serine [166] and the work in this issue on capped L-histidine, Nacetyl L-histidine N'-methylamide [237]. Of course there is much more work to do, extending to large systems, in even more complex environments, for example, embedded in membranes. In addition, multidimensional infrared and Raman techniques have recently been developed and multidimensional VCD has been proposed. In addition, surfaced enhanced Raman spectroscopy (SERS) has been extended to biological systems and even preliminary reports of surface enhanced Raman optical activity (SEROA). But the theory for these techniques still remained to be fully developed, tested and benchmarked, before these techniques become routinely used in molecular biophysics, like the other techniques covered in the recent Cambridge University Press book, Methods in Molecular Biophysics by Serdyuk, Zaccai and Zaccai [238]. In addition the recently developed TD-DFTB methods have been used to analyze the EA, ECD spectra of biomolecules [239,240], in addition to utilizing wave function based methods [241,242]. With the experimental Synchrotron Radiation Circular Dichroism (SRCD) technique now available for making measurments even farther in the UV range, clearly the tools of TD-DFT and wave function basd quantum mechancs have a lot of offer in the understanding and interpretiton of the exciting new experimental results [243]. In addition, some non linear optical methods have been proposed [244] and a general perspective paper on molecules in both electric and magnetic fields has appeared [245]. The effects of the solvent on vibrational frequencies have also recently been investigated and a new partitioning of the contributions causing the solvent effects has been proposed [246]. So many effects which were thought to be well 
Role of quantum chemical calculations in biophysics with a historical perspective

understood, both experimentally and theoretically, are being restudied and reinvestigated. This has been due to higher resolution and more accurate experimental data, which has pointed out some of the inadequacies of the previous theoretical descriptions. The articles in this special issue of TCA cover the areas of molecular biophysics, biophysical chemistry, chemical physics, mathematical biology, chemistry and physics and bioinformatics, many citing the cutting edge contributions to these fields by Professor Suhai, his group and large number of collaborators. We want to take this opportunity to thank once again Professor Sandor Suhai for his many contributions to these fields, for his educating and mentoring many BSc, MSc, PhD and postdoctoral students. He has been one of the leaders in these fields and with this special focus issue, all of the contributors have contributed what many feel are their best works in the past years. What better place to publish them than in this special focus issue of TCA.

With the passage of time the number of 'quantized' processes which have been investigated has increased and is continuing to increase. The rejection neuromediator at the generation of postsynaptic potentials, the formation of the action potential, and the investigation of the repair mechanism by the photolyase class of proteins in the presence of blue light are just three examples of problems which require further investigations. The later biological repair process of DNA is initiated by the absorption of a photon of light. Hence the process starts on the excited state energy in a very complex environment and evolves in time $[247,248]$. Clearly all of the tools and methods discussed in this review and covered in this special focus issue are needed to attack such problems. With this review we have tried to bring you to the point where you can now read the many research articles which follow which have been submitted from top research laboratories and groups on all five continents working the multidisciplinary fields of molecular biophysics, biophysical chemistry, chemical physics, mathematical biology, chemistry and physics, and bioinformatics.

\section{Acknowledgment}

AKK would like to thank all those who directly or indirectly contributed to this material. It is difficult to list all the names and I confine myself to expressing gratitude to my colleagues: S.A. Khuznetsova, S.V. Poltev, R.V. Belousov, and A.S. Rychkova. AKK is indebted to Dr. K. J. Jalkanen for the invitation to coauthor this manuscript with him on the occasion of Prof. Sandor Suhai's $65^{\text {th }}$ birthday and his, Professor Suhai's, many contributions to molecular biophysics. KJJ would like to thank the German Cancer Research Center (DKFZ) in Heidelberg, Germany and Professor Sandor Suhai, Head of the Department of Molecular Biophysics, for his initial invitation to come to the DKFZ in the fall of 1994 from the National Center for Supercomputing Applications (NCSA) on the campus of the University of Illinois at Urbana/Champaign (UIUC), for introducing him to the field of molecular biophysics, and finally for the 3 month stay at the DKFZ in Heidelberg in 2009 (April to June 2009). KJJ would also like to thank Professor Thomas Frauenheim and Dr. Thomas Niehaus for the visiting research scientist position at the Bremen Center for Computational Materials Science (BCCMS) at the University of Bremen for the period of Oct. 2008 to March 2009 and my good Ukrainian friend and colleague Dr. Grygoriy Dolgonos for critical reading of our manuscript and valuable discussions on DFT and WFQM methods as applied to calculations for biomolecules. KJJ would also like to thank Curtin University of Technology for providing infrastructure support for the period of October 2008 to July 2009, during which time KJJ was an Adjunct Research Fellow at the Nanochemistry Research Institute (NRI). The DFG and the DAAD are also acknowledged for financial support for KJJ's stay in Bremen and in Heidelberg during which time this review was coauthored with AKK. Finally I would like to thank Professor Victor I. Danilov for sending me reprints of his very good papers, some of which are only available in the Russian literature. 
Role of quantum chemical calculations in biophysics with a historical perspective

\section{References}

1. Bohr N (1933) Nature 131:421

2. Bohr N (1958) Atomic Physics and Human Knowledge, Wiley, New York

3. Schrödinger E (1945) What is Life? The Physical Aspects of the Living Cells, McMillan, New York

4. Weissbluth M, Ed. (1964) Quantum Aspects of Polypeptides and Polynucleotides, Biopolymers Symposia No.1. John Wiley \& Sons, Inc., New York

5 . Timoféeff-Ressovsky NW, Zimmer KG and Delbrück M (1935) Nachrichten von der Gesellschaft der Wissenschaften zu Göttingen 1:189

6. Frank J and Teller E (1938) J Chem Phys 6:861

7. Jordan P (1938) Naturwissenschaften 26:693

8. Pauling L and Corey RB (1951) Nature 168:550

9. Watson JD and Crick FHC (1953) Nature 171:737

10. Franklin RE and Gosling RG (1953) Nature 171:740

11. Franklin RE and Gosling RG (1953) Nature 172:156

12. Szent-Gyorgui A (1941) Nature 148:157

13. Petrov EG (1984) Fizika perenosa zaryadov v biosystemakh, Naukova Dumka, Kiev (in Russian).

14. Evans MG and Gergely J (1949) Biochim Biophys Acta 3:188

15. Pullman B and Pullman A (1963) Quantum Biochemistry, Wiley, New York.

16. Marois M, Ed. (1969) Theoretical Physics and Biology, Proceedings of the First International Conference on Theoretical Physics and Biology, Versailles, 26-30 June 1967. North-Holland Publ. Company, Amsterdam and London

17. Moffit W (1956) J Chem Phys 25:467

18. Davydov AS (1951) Teoriya pogloshcheniya sveta v molekulyarnykh kristallakh, Akad. Nauk USSR, Kiev (in Russian)

19. Yomosa S (1964) "П-electronic Structure of Peptide Group and Hydrogen-Bonded Polypeptides," in Quantum Aspects of Polypeptides and Polynucleotides, Biopolymers Symposia No. 1, p. 1-33. M. Weissbluth, Ed. John Wiley \& Sons, Inc., New York

20. Suard MG, Berthier G and Pullman B (1961) Biochim Biophys Acta 52:254

21. Cardew MH and Eley DD (1959) Discus. Farad. Soc. 27:115

22. Chance, B. (1961) "Free radicals and enzyme-substrate compounds," in Proceedings of the Symposium on Free Radicals in Biological Systems, Stanford University, 1960, p. 1-16. M.S. Blois, H.W. Brown, R.M. Lemmon, R.O. Lindblom, and M. Weissbluth, Eds. Academic Press, New York and London

23. Commoner, B., Townsend J. and Pake, G.E. (1954) Nature 174:689

24. Gordy W, Ard WB and Shields H (1954) Proc. Natl. Acad. Sci. USA 41:983

25. Ehrenberg A (1957) Acta Chem Scand 11:205 
Role of quantum chemical calculations in biophysics with a historical perspective

26. Beinert H and Sands RH (1959) Biochem Biophys Res Comm 1:171

27. Calvin M and Sogo PB (1957) Science 125:499

28. Sogo PB, Pon NG and Calvin M (1957) Proc. Natl. Acad. Sci USA 43:387

29. Sogo PB, Jost M and Calvin M (1959) Radiation Rev. (Suppl.) 1:511

30. Arnold W and Clayton RR (1960) Proc. Natl. Acad. Sci. USA 46:776

31. Chance B and Nishimura M (1960) Proc. Natl. Acad. Sci. USA 46:19

32. De Vault D and Chance B (1966) Biophys J 6:825

33. Grigorov LN and Chernavsky DS (1972) Biofizika 17:95 (in Russian)

34. Chernavskaya NM and Chernavsky DS (1973) Studia Biophysica 35:149

35. Hopfield JJ (1974) Proc. Natl. Acad. Sci. USA 71:3640

36. Jortner J (1976) J. Chem. Phys. 64:4860

37. Ulstrup J and Jortner J (1975) J Chem Phys 63:4358

38. Bixon M and Jortner J (1968) J Chem Phys 48:715

39. Dogonadze RR, Kuznetsov AM and Ulstrup J (1977) J Theor Biol 69:239

40. Kharkyanen VN, Petrov EG and Ukrainskii II (1978) J Theor Biol 73:29

41. Sarai A (1980) Biochim Biophys Acta 589:71

42. Jortner J (1980) Biochim Biophys Acta 594:193

43. Joliot P, Barbieri G and Chabaud R (1969) Photochem Photobiol 10:309

44. Joliot P, Joliot A, Bouges B and Barbieri, G (1971) Photochem Photobiol 14:287

45. Kok B, Forbush B and McGloin M (1970) Photochem Photobiol 11:457

46. Shuvalov VA and Klimov VV (1976) Biochim. Biophys. Acta 440:587

47. Shuvalov VA, Sharkov AV, Matvets YA and Krukov PG (1978) FEBS Lett. 91:135

48. Deisenhofer J, Epp O, Miki K, Huber R and Michel H (1984) J Mol Biol 180:385

49. Woodbury NW, Becker M, Middendorf D and Parson WW (1985) Biochem 24:7516

50. Martin J-L, Breton J, Hoff AJ, Migus A and Antonetti A (1986) Proc. Natl. Acad. Sci. USA 83:937

51. Breton J, Martin J-L, Fleming RG and Lumbry JC (1988) Biochem 27:8276

52. Vos M, Rappaport F, Lumbry J-C, Breton J and Martin J-L (1993) Nature 363:320

53. Vos M, Jones MR, Hunter CN, Breton J and Martin J-L (1994) Proc. Natl. Acad. Sci. USA. 91: 12701

54. Vos MH, Jones MR, Breton J, Lumbry JC and Martin J-L (1996) Biochem 35:2687

55. Bixon J, Jortner J, Michel-Beyerle ME and Ogrodnik A (1989) Biochim Biophys Acta 977:273

56. Michel-Beyerle ME, Plato M, Deisenhofer J, Michel H, Bixon J and Jortner J (1988) Biochim Biophys Acta 932:52 
Role of quantum chemical calculations in biophysics with a historical perspective

57. Marcus RA and Sutin N (1985) Biochim Biophys Acta (Reviews on Bioenergetics) 811:265

58. Parson WW, Chu Z-T and Warshel A (1990) Biochim Biophys Acta (Bioenergetics) 1017:251

59. Creighton S, Hwang J-K, Warshel A, Parson WW and Norris J (1988) Biochem 27:774

60. Zhang WM, Meier T, Cherniak V and Mukamel S (1988) J Chem Phys 108:7763

61. Shuvalov VA (2000) Preobrazovanie solnechnoi energii v pervichnom akte razdeleniya zaryadov v reaktsionnykh tsentrakh fotosinteza (Conversion of Solar Energy in a Primary Act of Charge Separation in Photosynthetic Reaction Centers), Nauka, Moscow (in Russian).

62. Suard M (1962) Biochim Biophys Acta 59:227

63. Suard M (1962) Biochim Biophys Acta 64:400

64. Roothaan CCJ (1951) Rev Mod Phys 23:69

65. Pariser R. and Parr R.G. (1953) J Chem Phys 21:466

66. Löwdin P.O. (1950) J Chem Phys 18:365

67. Ham SS and Platt JR (1952) J Chem Phys 20:335

68. Lyons LE (1957) J Chem Soc 5001

69. Blumenfeld LA and Benderski VA (1963) J Struct Chem 4:415 (in Russian)

70. Kukushkin AK (1970) Vestnik Mosk. Gos. Univ., Ser. Fiz., Astron. 2:205 (in Russian)

71. Kukushkin AK and Tikhonov AN (1988) Lectures on biophysics of plant photosynthesis, Moscow State University Publ., Moscow

72. Tinoco I, Jr (1960) J Am Chem Soc 82:4785; Erratum: (1960) J Am Chem Soc 83:5047

73. Rhodes W (1961) J Am Chem Soc 83:3609

74. DeVoe H (1964) Quantum Aspects of Polypeptides and Polynucleotides, Biopolymers Symposia No. 1, p. 251. M. Weissbluth, Ed. John Wiley \& Sons, Inc., New York

75. Nesbet RK (1962) Mol Phys 5:63

76. Bolton HC and Weiss JJ (1962) Nature 195:666

77. Kirkwood JG (1937) J Chem Phys 5:479

78. Tinoco I, Jr (1960) J Chem Phys 33:1332; Erratum: (1961) J Chem Phys 34:1067

79. Pullman B and Pullman A (1958) Bull Soc Chim 766 (in French)

80. Veillard A and Pullman B (1963) J Theor Biol 4:37

81. Pariser R and Parr RG (1953) J Chem Phys 21:466

82. Pople JA (1953) Trans Farad Soc 49:1375

83. Bradley DF, Lifson S and Honig B (1964) Electronic Aspects of Biochemistry, B Pullman, Ed. Academic Press, New York, 77

84. Del Re G (1958) J Chem Soc 4031

85. Rein R, Fukyda N, Clarke GA and Harris FE (1968) J Theor Biol 21:88 
Role of quantum chemical calculations in biophysics with a historical perspective

86. Pullman B and Pullman A (1959) Biochim Biophys Acta 36:343

87. Hoffmann TA and Ladik J (1964) Adv Chem Phys 7:84

88. Danilov VI, Kuprievich VA and Shramko OV (1967) Biofizika 12:186 (in Russian)

89. Danilov VI (1967) Biofizika 12:540 (in Russian)

90. Danilov VI, Shramko OV and Dyadusha GG (1967) Biofizika 12:544 (in Russian)

91. Danilov VI, Kuprievich VA and Shramko OV (1967) Biofizika 12:606 (in Russian)

92. Kuprievich VA, Danilov VI and Shramko OV (1967) Mol Bio 1:343 (in Russian).

93. Danilov VI, Kuprievich VA and Shramko OV (1967) Doklady Akademii Nauk 177:1465 (in Russian).

94. Löwdin PO . (1964) Quantum Aspects of Polypeptides and Polynucleotides, Biopolymers Symposia No. 1, p. 61. M. Weissbluth, Ed. John Wiley \& Sons, Inc., California

95. Beukers R and Berends W (1960) Biochim Biophys Acta 38:573

96. Pulman A (1969) Annals New York Academy of Sciences, 158:65-85

97. Lewin B (1991) Gene, Lehrbuch der molekularen Genetik, Zweite Auflage, VCH, Weinheim, p 65

98. Kruglyak YA, Danilov VI, Kuprievich VA and Ogloblin VV (1972) Theor Exper Chem 6:28-33 (in English)

[(1970) Teoreticheskaya I Eksperimental'naya Khimiya 6:33-39 in Russian]).

99. Löwdin PO (1963) Rev Mod Phys 35:724

100. Ladik J (1964) J Theor Biol 6:201

101. Rein R and Ladik J (1964) J Chem Phys 40:2466

102. Rein R and Harris FE (1964) J Chem Phys 41:3393

103. Rein R and Harris FE (1965) J Chem Phys 43:4415

104. Clementi E, Mehl J and von Niessen W (1971) J Chem Phys 54:508

105. Blizzard AC and Santry DP (1969) J Theor Biol 25:461

106. Maranon J, Sorarrain OM, Grinberg H, Lamdan S and Gaozza CH (1978) J Theor Biol 74:11

107. Merchan M and Serrano-Andres L (2003) J Am Chem Soc 125:8108

108. Serrano-Andres L and Merchan M (2005) J Molec Struct (THEOCHEM) 729:109

109. Poltev VI and Shulyupina NV (1984) Mol Biol 18:154 (in Russian).

110. Poltev VI (1983) Structural and functional aspects of DNA replication and reparation. AI Gaziev, Ed. Institute of Biophysics, Puschino, 101 (in Russian).

111. Berthod H and Pullman AJ (1965) J Chim Phys 62:942 (in French)

112. Hirata S, Ivanov S, Grabowski I, Barlett RJ, Burke K and Talman JD (2001) J Chem Phys 115:1635

113. Poltev VI, Shulyupina NV and Bruskov VI (1980) Studia Biophysica 79:45

114. Chuprina VP, Khutorsky VE and Poltev VI (1981) Studia Biophysica 85:81

115. Poltev VI, Chuprina VP, Shulyupina NV and Bruskov VI (1982) Studia Biophysica 87:247 
Role of quantum chemical calculations in biophysics with a historical perspective

116. Chuprina VP and Poltev VI (1983) Nucl Acids Res 11:5205

117. Hug V and Tinoco I, Jr (1973) J Am Chem Soc 95:2803

118. Pullman A (1968) Int J Quantum Chem, Symp 2:187

119. Snyder LC, Shulman RG and Neumann DB (1970) J Chem Phys 53:256

120. Aida M, Negishi K, Hayatsu H and Maeda M (1988) Biochem Biophys Res Comm 153:552

121. Tarashima S (1969) Biopolymers 8:199

122. DeVoe H and Tinoco I, Jr (1962) J Mol Biol 4:500

123. Rein R and Pollak M (1967) J Chem Phys 47:2039

124. Eley DD and Spivey DI (1962) Trans Faraday Soc 58:411

125. O’Konski CT, Moser P and Shirai M (1964) Biopolymers Symp. 1:479

126. Sobti NK and Pant MM (1975) Indian J Biochem Biophys 12:216

127. Warman JM, De Haas MP and Rupprecht A (1996) Chem Phys Lett 249:319

128. Olson EJC, Hu D, Hörmann A and Barbara PF (1997) J Phys Chem B 101:299

129. Dandliker PL, Holmlin RE and Barton JK (1997) Science $275: 1464$

130. Oganesyan VS, Hubbard PL, Butt JN and Jayasooriya UA (2003) Physica B: Condensed Matter 326:25

131. Lange AW and Herbart JM (2008) Abstracts of OSU International Symposium on Molecular Spectroscopy, URI: http://hdl.handle.net/1811/33397

132. Otten HA (1971) Photochem. Photobiol 14:289

133. Nagae H and Yomosa S (1977) J Phys Soc Japan. 42:998

134. Knop JV and Stichtenoth H (1972) Z Naturforsch. 27A:1972

135. McHugh AJ, Gouterman M and Weiss C, Jr (1972) Theor Chem Acc 24:346

136. Maggiora GM and Weimann LJ (1974) Int J Quantum Chem: Quantum Biol Symp 1:179

137. Yip KL, Duke CB, Salaneck WR, Plummer EW and Loubriel G (1977) Chem Phys Lett 49:530

138. Petke JD, Maggiora GM, Shipman LL and Christoffersen RE (1978) J Mol Spectros 73:311

139. Petke JD, Maggiora GM, Shipman LL and Christoffersen RE (1980) Photochem Photobiol 31:243

140. Petke JD, Maggiora GM, Shipman LL and Christoffersen RE (1979) Photochem Photobiol 30:203

141. Petke JD, Maggiora GM, Shipman LL and Christoffersen RE (1980) Photochem Photobiol 32:399

142. Loach PA, Bambara RA and Ryan RJ (1971) Photochem Photobiol 13:247

143. Norris JR, Uphaus RA and Katz JJ (1971) Proc Natl Acad Sci USA 68:625

144. Petke JD, Maggiora GM, Shipman LL and Christoffersen RE (1978) J Mol Spectrosc 71:64

145. Whitten JL and Hackmeyer M (1969) J Chem Phys 51:5584

146. Christoffersen RE (1972) Adv Quantum Chem 6:333 
Role of quantum chemical calculations in biophysics with a historical perspective

147. Christoffersen RE, Spangler D, Hall GG and Maggiora GM (1973) J Am Chem Soc 95:8526

148. Spangler D, Maggiora GM, Shipman LL and Christoffersen RE (1977) J Am Chem Soc 99:7470

149. Spangler D, Maggiora GM, Shipman LL and Christoffersen RE (1977) J Am Chem Soc 99:7478

150. Maslov VG (1981) Theoretical and Experimental Chemistry 17:372 (in Russian).

151. Plato M, Trankle E, Lubitz W, Lendzian F and Mobius K (1986) Chem Phys 107:185

152. Sinnecker S, Koch W and Lubitz W (2002) J Phys Chem B 106:5281

153. Berezin KV, Nechaev VV and Ziganshina OD (2004) J Struct Chem 45:217

154. Berezin KV and Nechaev VV (2005) J Appl Spectros 72:164.

155. Eriksson LA, Himo F, Siegbahn PEM and Babcock GT (1997) J Phys Chem A 101:9496

156. O'Malley PJ (1999) Biochim Biophys Acta 1411:101

157. Rinyu L, Nagy L and Kortvelyesi T (2001) J Mol Struct (Theochem) 571:163

158. Ikryannikova LN, Ustynyuk LY and Tikhonov AN (2004) J Phys Chem A 108:4759

159. Perdew JP, Burke K and Ernzerhof M (1996) Phys Rev Lett 77:3865; Errata: (1997) Phys Rev Lett 78:1396.

160. Dittrich M, Freddolino PL and Schulten K (2005) J Phys Chem B 109:3006

161. Jalkanen, KJ (2003) J. Phys: Condens. Matter 15:S1823

162. Jalkanen KJ, Elstner M and Suhai S (2004) J Mol Struct (Theochem) 675:61

163. Jalkanen KJ, Nieminen RM, Frimand K, Bohr J, Bohr H, Wade RC, Tajkhorshid E and Suhai S (2001) Chem Phys 265:125

164. Han W-G, Elstner M, Jalkanen KJ, Frauenheim T and Suhai S (2000) Int J Quantum Chem 78:459

165. Bohr H and Jalkanen KJ (2005) Condensed Matter Theory, JW Clark, RM Panoff and H Li, Ed. Nova Science Publishers, Hauppauge, NY, 20:375

166. Jürgensen VW and Jalkanen KJ (2006) Phys Biol 3:S63

167. Jalkanen KJ, Nieminen RM and Bohr J (2000) Vestnik Moskovskogo Universiteta. Khimiya 41:4 (Proceedings of the International Conference BIOCATALYSIS-2000: FUNDAMENTALS \& APPLICATIONS Moscow, Russia, June 10-15, 2000) Journal link: [http://www.chem.msu.su/eng/journals/vmgu/00add/welcome.html] PDF link: [http://www.chem.msu.su/eng/journals/vmgu/00add/4.pdf]

168. Abdali S, Jalkanen KJ, Cao X, Nafie LA and Bohr H (2004) Phys Chem Chem Phys 6:2434

169. Kuhn H (1986) Phys Rev A 34:3409

170. Datta SN and Mallik B (1997) Int J Quantum Chem 61:865

171. Shipman LL (1982) Photosynthesis, Volume 1, Energy conversion by plants and bacteria, p. 403.

Govingjee, Ed. Academic Press, New York, London

172. Bylina EJ, Kirmaier C, Mc Dowell L, Holten D and Youvan DC (1988) Nature 336:182

173. Sadygov RG and Kukushkin AK (1991) Biofizika 36:990 (in Russian). 
Role of quantum chemical calculations in biophysics with a historical perspective

174. Parush OV, Sadygov RG and Kukushkin AK (1994) Biofizika 39:848 (in Russian).

175. Mataga N (1958) Bull Chem Soc Japan 31:453

176. Xei XL and Simon JD (1991) Biochim Biophys Acta 1057:131

177. Deisenhofer J, Epp 0 and Miki R (1985) Nature 318:618.

178. Jortner J (1980) Biochim Biophys Acta 594:193

179. Sakuma T, Kashiwagi H, Takada T and Nakamura H (1997) Int J Quantum Chem 61:137

180. Peluso A, Di Donato M and Saracino GA (2000) J Chem Phys 113:3112

181. Wu SW, Ogawa N and Ho W (2006) Science 312:1362

182. Damjanovic A, Kosztin I, Kleinekathofer U and Schulten K (2002) Phys Rev E 65:031919

183. Kukushkin AK, Poltev S and Khuznetsova S (2002) Bioelectrochem 56:9

184. Tomchuk P, Procenko N and Krasnogolovets V (1985) Biochim Biophys Acta 807:272

185. Belousov RV, Poltev SV and Kukushkin AK (2003) Biophysics 40:606

186. Dewar MJS and Thiel W (1977) J Am Chem Soc 99:4899

187. Ziganshina OD (2005) The quantum mechanical calculations of the vibrational spectra and structure of cofactors of photosynthtical reaction center. Ph. D. Thesis, N.G. Chernyshevski Saratov State University Press, Saratov (in Russian)

188. Belousov RV, Belov AA and Kukushkin AK (2004) Biofizika 49:463 (in Russian)

189. Krasilnikov PM and Mamonov PA (2006) Biofizika 51:267 (in Russian)

190. Tulub AA, Stefanov VE and Skaletskii EK (2001) Biofizika 46:581 (in Russian)

191. Green ME (2001) J Phys Chem A 105:5298

192. Sapronova A, Bystrov V and Green ME (2003) J Mol Struct (Theochem) 630:297

193. Ben-Nun M, Molnar F, Lu H, Phillips JC, Martinez TJ and Schulten K (1998) Faraday Discuss 110:447

194. Hayashi S, Tajkhorshid E, Kandori H and Schulten K (2004) J Am Chem Soc 126:10516

195. Hayashi S and Ohmine I (2000) J Phys Chem B 104:10678

196. Grigorenko BL, Nemukhin AV, Shadrina M, Topol I and Burts S (2007) Proteins 66:456

197. Nemukhin AV, Grigorenko BL and Shadrina M (2007) Ros. Khim. Zh. 51:27 (in Russian).

198. Nemukhin AV, Grigorenko BL and Granovski AA (2004) Vestnik Moscow University, ser. 2, chemistry, 45:75 (in Russian) [http://www.chem.msu.su/rus/vmgu/042/abs001.html]

199. Nemukhin AV, Bochenkova AV, Bravaya KV and Granovsky AA (2006) Proc. of SPIE 6449, 6449N(1)

200. Bravaya KV, Bochenkova AV, Granovsky AA and Nemukhin AV (2007) J Am Chem Soc 129:13035

201. Bravaya KV, Bochenkova AV,Granovsky AA, Savitsky AP and Nemukhin AV (2008) J Phys Chem A 112:8804

202. Masgrau L, Roujeinikova A, Johannissen LO, Hothi P, Basran J, Ranaghan KE, Mulholland AJ, Sutcliffe MJ, Scrutton NS and Leys D (2006) Science 312:237 
Role of quantum chemical calculations in biophysics with a historical perspective

203. Rovira C (2003) J Phys: Condens Matter 15:S1809

204. Car R and Parinello M (1985) Phys Rev Lett 55:2471

205. Marx D, Tuckerman M and Martuna GJ (1999) Comput Phys Comm 118:166

206. Kusunoki M, Kitaura K, Morokuma K and Nagata C (1980) FEBS Lett 117:179

207. Zouni A, Witt HT, Kern J, Fromme P, Krauss N and Saenger W (2001) Nature 409:739

208. Kamiya N and Shen JR (2003) Proc Natl Acad Sci USA 100:98

209. Ferreira KN, Iverson TM, Maghlaoui K, Barber J and Iwata S (2004) Science 303:1831

210 . Raval MK and Biswal UC (1985) J Theor Biol 115:137

211. Denisenko AS and Kukushkin AK (2005) Biofiziki 50:833 (in Russian)

212. Kusunoki M (2006) Programme and Abstracts of International Meeting "Photosynthesis in the PostGenomic Era: Structure and Function of Photosystems", p. 104. I.I.Proskuryakov, Ed. NIA-Priroda, Moscow.

213. Sproviero EM, Gascon JA, McEvoy JP, Brudvig GW and Batista VB (2006) J Chem Theory Comput 2:1119

214 . Filatov M and Cremer D (2005) J Chem Phys 123:124101

215. Stephens PJ, Devlin F, Chabalowski CF and Frisch M (1994) J Phys Chem 98:11623

216. Runge E and Gross EKU (1984) Phys Rev Lett 52:997

217. Wanko M, Garavelli M, Bernardi F, Niehaus TA, Frauenheim T and Elstner M (2004) J Chem Phys 120:1674

218. Young DC (2001) Computational Chemistry: A Practical Guide for Applying Techniques to Real-World Problems, John Wiley \& Sons, Inc., New York, New York USA

219. Koch W and Holthausen MC (2000) A Chemist's Guide to Density Functional Theory Wiley-VCH, Weinheim, Germany

220. Parr RG and Yang W (1989) Density-Functional Theory of Atoms and Molecules, Oxford University Press, Oxford, UK

221. Frauenfelder H, Wolynes PG and Austin RH (1999) Rev Mod Phys 71:419

222. Segall MD 2002, J Phys: Condens Matter 14:2957

223. Stephens PJ and Devlin FJ (2007) "Vibrational Circular Dichorism”, in Continuum Solvation Models in Chemical Physics: From Theory to Applications, pp. 180-205. B. Mennucci and R. Cammi, Eds. John Wiley \& Sons, Inc., New York

224. Jalkanen KJ, Bohr HG and Suhai S (2009) "Quantum Molecular Biological Methods Using Density Functional Theory," in Handbook of Molecular Biophysics, pp. 7-66. H.G. Bohr, Ed. Wiley-VCH Verlag GmbH \& Co. KGaA, Weinheim, Germany.

225. Jalkanen KJ and Suhai S (1996) Chem Phys 208:81

226. Deng Z, Polavarapu PL, Ford SJ, Hecht L, Barron LD, Ewig CS and Jalkanen KJ (1996) J Phys Chem 100:2025

227. Jalkanen K.J., Bohr H. and Suhai S. (1997) 'Density functional and neural network analysis: hydration effects and spectroscopic and structural correlations in small peptides and amiono acids' in Theoretical and 
Role of quantum chemical calculations in biophysics with a historical perspective

Computational Methods in Genome Research, pp. 255-277. S Suhai, Ed. Plenum Press, New York, New York USA

228. Han W, Jalkanen KJ, Elstner M and Suhai S (1998) J Phys Chem B 102:2587

229. Bohr H, Jalkanen KJ, Frimand K, Elstner M and Suhai S (1999) Chem Phys 246:13

230. Jalkanen KJ, Degtyarenko IM, Nieminen RM, Cao X, Nafie LA, Zhu F, and Barron LD (2008) Theor Chem Acc 119:191

231. Tajkhorshid E, Jalkanen KJ and Suhai S (1998) J Phys Chem B 102:5899

232. Frimand K, Jalkanen, KJ, Bohr HG and Suhai S (2000) Chem Phys 225:165

233. Abdali S, Jalkanen KJ, Bohr H, Suhai S and Nieminen RM (2002) Chem Phys 282:219

234. Knapp-Mohammady M, Jalkanen KJ, Nardi F, Wade RC and Suhai S (1999) Chem Phys 240:63

235. Jalkanen KJ, Nieminen RM, Knapp-Mohammady M and Suhai S (2004) Int J Quantum Chem 92:239

236. Deplazes E, van Bronswijk W, Zhu F, Barron LD, Ma S, Nafie LA and Jalkanen KJ (2008) Theor Chem Acc 119:155

237. Jalkanen KJ, Ma D, Nafie LA, Zhu F, Barron LA, Aoki Y, Rohl AL and Gale JD (2009) Theor Chem Acc (this issue)

238. Serdyuk IN, Zaccai NR and Zaccai J (2007) Methods in Molecular Biophysics, Cambridge University Press, Cambridge, UK

239. Tanaka T, Kodama TS, Morita HE and Ohno T (2006) Chirality 18:652

240. Sebek J, Gyurcsik B, Sebestik J, Kejik Z, Bednarova L and Bour P (2007) J Phys Chem A 111:2750

241. Rogers DM and Hirst JD (2004) Biochem 43:11092

242. Oakley MT and Hirst JD (2006) J Am Chem Soc 128:12414

243. Miron S, Refregiers M, Gilles A-M and Maurizot J-C (2005) Biochem Biophys Acta 1724:425

244. Tabisz GC, Cameron R and Qu W (2008) Annu Rep Prog Chem C 104:13

245. Buckingham AD (1998) Annu Rev Phys Chem 49:xiii

246. Varfolomeev MA, Abaidullina DI, Klimovitskii AE and Solomonov BN (2007) Russ J Gen Chem 77:1742 [also in Russian, (2007) Zhurnal Obshchei Khimii 77:1677]

247. Bohr H, Jalkanen KJ and Malik FB (2005) Mod Phys Lett B 19:473

248. Jalkanen KJ, Jürgensen VW, Claussen A, Rahim A, Jensen GM, Wade RC, Nardi F, Jung C, Degtyarenko IM, Nieminen RM, Herrmann F, Knapp-Mohammady M, Niehaus TA, Frimand K and Suhai S (2006) Int J Quantum Chem 106:1160 
Role of quantum chemical calculations in biophysics with a historical perspective

Figure Legends:

Figure 1: Atom numbering for adenine-thymine (A-T) and guanine-uracil (Г-Ц), reproduced from reference [91] 


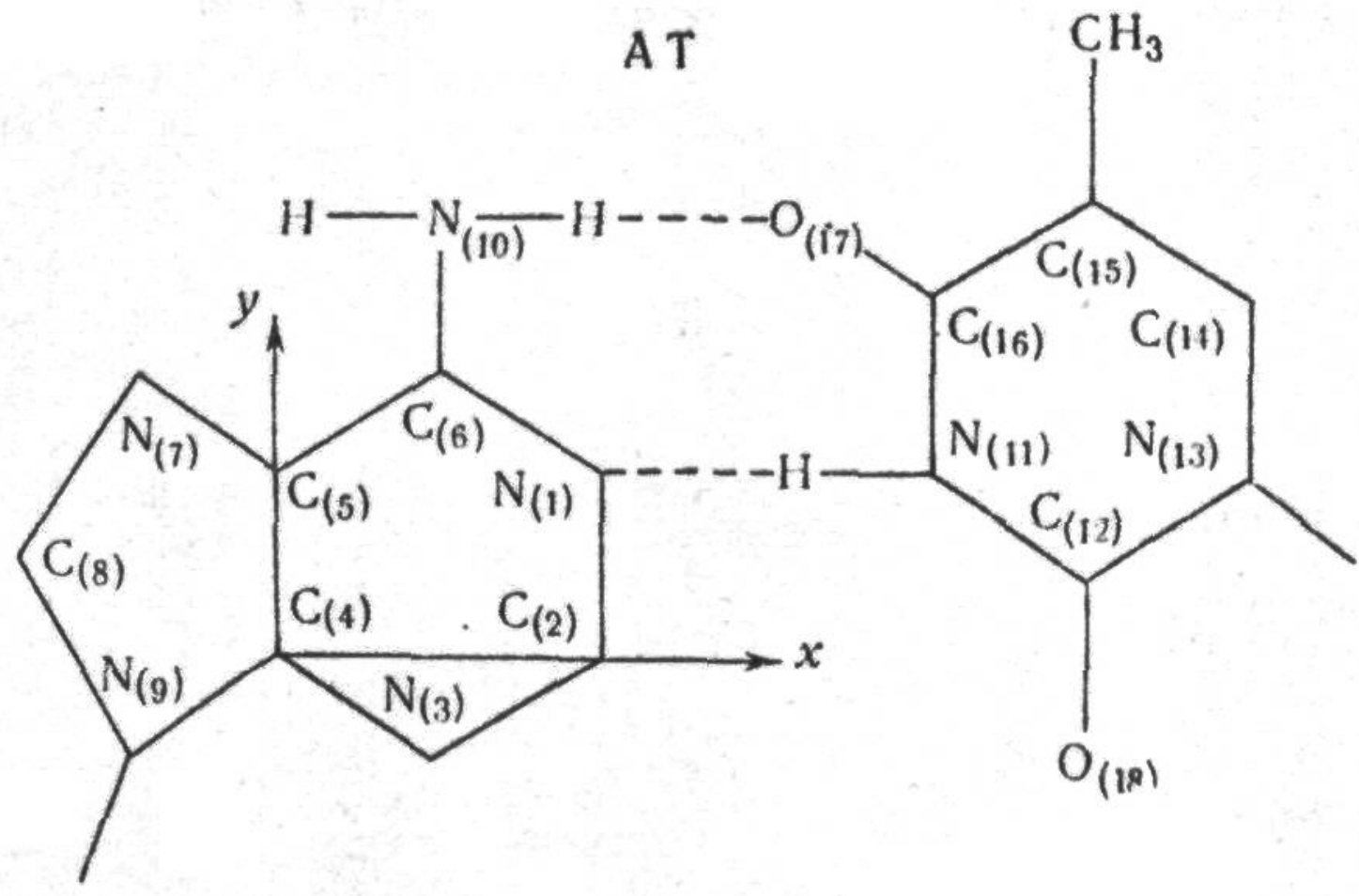

ru

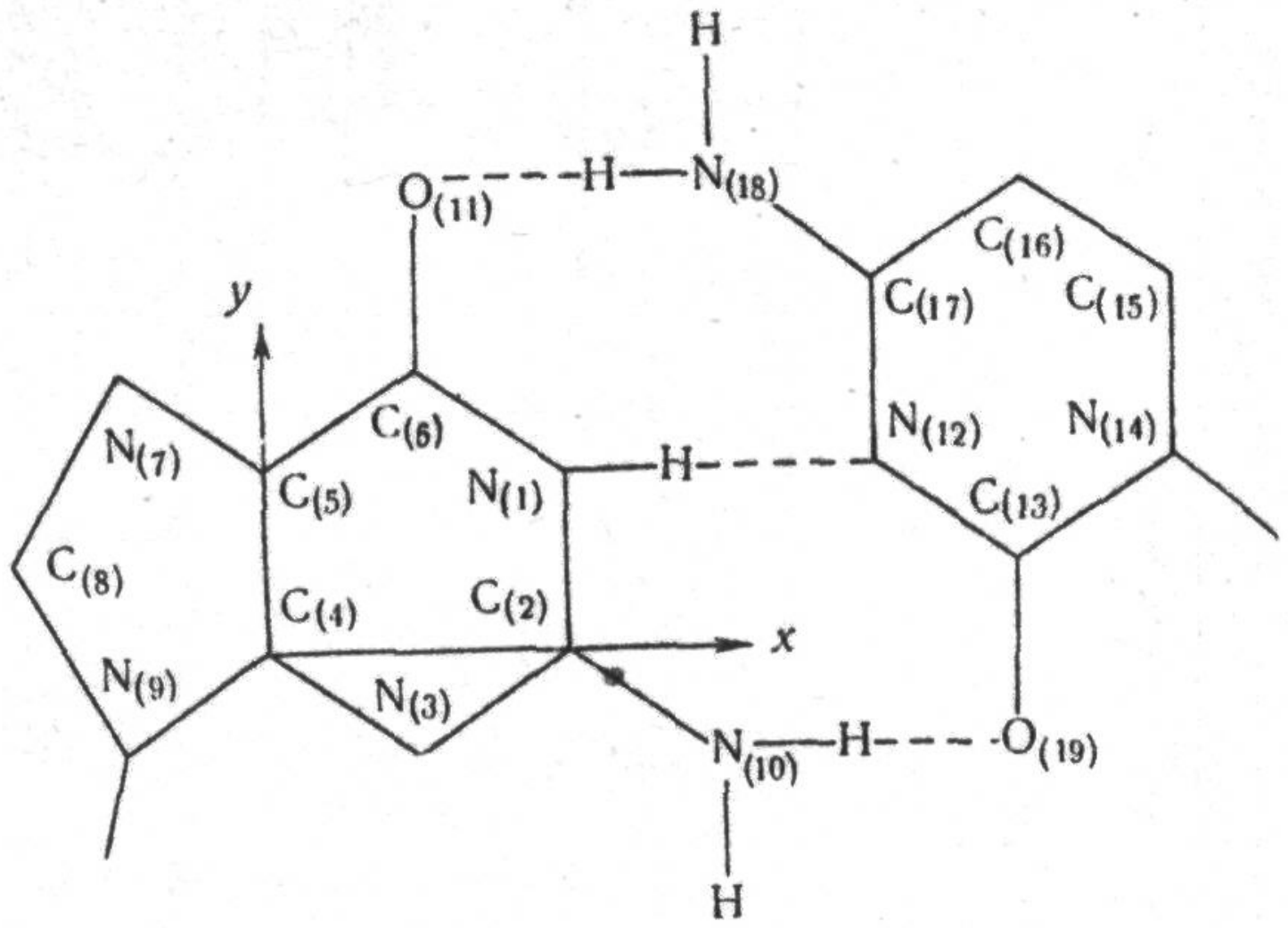

